\title{
Grape Sour Rot: A Four-Way Interaction Involving the Host, Yeast, Acetic Acid Bacteria, and Insects
}

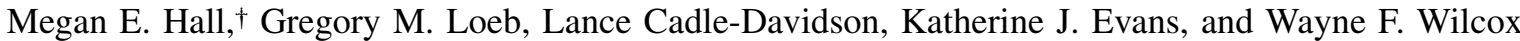

First and fifth authors: Section of Plant Pathology and Plant-Microbe Biology, School of Integrative Plant Science, and New York State Agricultural Experiment Station, Cornell University, Geneva 14456; second author: Department of Entomology, New York State Agricultural Experiment Station, Cornell University; third author: United States Department of Agriculture-Agricultural Research Service, Grape Genetics Research Unit, Geneva, NY 14456; and fourth author: Tasmanian Institute of Agriculture, University of Tasmania, Hobart, Tas. 7001, Australia.

Accepted for publication 28 June 2018.

\begin{abstract}
Sour rot, a disease affecting berries of cultivated Vitis spp. worldwide, has not been clearly defined. Reported symptoms of the disease include browning of the berry skin, oozing of disintegrated berry pulp, and the smell of acetic acid, all in the presence of fruit flies (Drosophila spp.). We determined acetic acid concentrations in multiple collections of symptomatic berries, isolated and identified microbes from them, and inoculated commonly isolated organisms into healthy berries with and without concurrent exposure to wild-type or axenic Drosophila melanogaster. Coinoculations combining one of several yeasts (Metschnikowia spp., Pichia spp., and a Saccharomyces sp.) plus

an acetic acid bacterium (an Acetobacter sp. and Gluconobacter spp.) reproduced sour rot symptoms, defined here as decaying berries with a loss of turgor and containing acetic acid at a minimum of $0.83 \mathrm{~g} / \mathrm{liter}$, based on observed field levels. Symptoms developed only in the presence of D. melanogaster, either wild type or axenic, indicating a nonmicrobial contribution of these insects in addition to a previously suggested microbial role. We conclude that sour rot is the culmination of coinfection by various yeasts, which convert grape sugars to ethanol, and bacteria that oxidize the ethanol to acetic acid, and that this process is mediated by Drosophila spp.
\end{abstract}

The etiology of sour rot, a disease affecting grape (Vitis spp.) in many temperate viticultural regions, is poorly understood. Disease symptoms are characterized by oxidation of the grape skin in which fruit of both red and white varieties turn brown, with the pulp oozing from the berries and smelling of acetic acid or (less commonly) ethyl acetate. Numerous fruit flies (or vinegar flies [Drosophila spp.]) are typically associated with the diseased fruit (McFaddenSmith and Gubler 2015). In some regions, workers have noted signs of mold growth on diseased fruit (Haviland et al. 2017; RooneyLatham et al. 2008), although such observations are far from universal. The development of sour rot symptoms can be extremely detrimental to the grape crop in affected vineyards. Infected clusters are often not harvested or are removed during postharvest sorting due to their unacceptability for fresh use or the increased risk of producing wine with unacceptable levels of total and volatile acidity (Barata et al. 2011).

There is disagreement within the published literature about the organisms causing sour rot and, consequently, what the appropriate control practices should be. Sour rot was originally thought to be the final stage of gray mold, caused by Botrytis cinerea (Bisiach et al. 1982, 1986). It is now accepted as a separate disease, although the term "sour rot" is still often applied to a general decay syndrome, which may involve filamentous fungi (Haviland et al. 2017; McFadden-Smith and Gubler 2015; Rooney-Latham et al. 2008). Several researchers have claimed that yeasts play an essential role in the development of sour rot (Barata et al. 2012a; Bisiach et al. 1982; Guerzoni and Marchetti 1987). Some also have noted the common

†Corresponding author: Megan E. Hall; E-mail: meh338@cornell.edu

Funding: This work was supported the New York State Department of Agriculture and Markets, New York Wine and Grape Foundation, Specialty Crops Research Initiative (grant C200834), and the Dyson Fund.

(c) 2018 The American Phytopathological Society association of acetic acid-producing bacteria (AAB) with the disease (Bisiach et al. 1986), whereas Barata et al. (2012a) concluded that $\mathrm{AAB}$ should be considered the etiological agents of sour rot.

The role of Drosophila fruit flies in the etiology of sour rot remains unclear. Guerzoni and Marchetti (1987) investigated the abundance of yeast associated with the disease while also noting the invariable presence of Drosophila spp. on rotten grape berries. Bisiach et al. (1986) concluded that controlling the disease could only be accomplished by managing Drosophila spp. or reducing berry wounds, believing that the insects served as necessary vectors of the causal organisms to these wounds. Barata et al. (2012a) showed that wounded berries did not develop sour rot when they were physically separated from Drosophila spp., and emphasized the presumed role of these insects as microbial vectors. They also noted that wounded berries did not heal when the flies were present, thereby remaining in a state susceptible to decay.

Given that previous reports do not present a consistent description of sour rot or its causes, the purpose of this research was to better define the symptoms, mechanism of symptom development, and etiology of this disease.

\section{MATERIALS AND METHODS}

Field samples. In 2013, we collected 16 clusters exhibiting visual and olfactory sour rot symptoms from each of 12 affected vineyards in the Finger Lakes region of New York (olfactory symptoms were those of acetic acid; ethyl acetate was not detected). Each cluster was placed individually into a low-density polyethylene bag (Fisher Scientific, Pittsburgh) and transported to the laboratory, where the entire cluster was macerated in the bag; then, the juice was extracted into a 50-ml Falcon tube and centrifuged at $4,000 \times g$ for $10 \mathrm{~min}$. Supernatant $(1 \mathrm{ml})$ from each sample was transferred into a $1.5-\mathrm{ml}$ collection tube, centrifuged at $10,000 \times g$ for $10 \mathrm{~min}$, and diluted twofold in water prior to filtration through a 
0.2- $\mu \mathrm{m}$ polyethersulfone (PES) membrane (Krackeler Scientific, Inc., Albany, NY) and immediate high-performance liquid chromatography (HPLC) analysis for ethanol and acetic acid concentration, as described below. In 2014 and 2015, four similarly affected clusters were collected from each of seven and nine vineyard blocks, respectively, sampled in the Finger Lakes region. Whole clusters were bagged individually and transported to the lab, where three symptomatic berries from each of four clusters per vineyard were detached by cutting, with surface-sterilized scissors, at the point where the pedicel is attached to the rachis. One berry was sampled from the tip of the cluster and one each from the anterior and posterior sides near the cluster middle. The three-berry samples were macerated in plastic bags and the juice was extracted into 1.5-ml collection tubes. The juice was then homogenized for $30 \mathrm{~s}$ using a vortex mixer and centrifuged at $10,000 \times g$ for $10 \mathrm{~min}$. The supernatant was transferred into clean collection tubes and stored at $-4^{\circ} \mathrm{C}$ until use, when the clarified juice samples were thawed at room temperature for $30 \mathrm{~min}$ and diluted twofold in water prior to filtration through a $0.2-\mu \mathrm{m}$ PES membrane and immediate HPLC analysis for ethanol and acetic acid content thereafter. Three additional symptomatic berries were similarly sampled from each cluster in 2014 and 2015 and used for isolation and subsequent identification of sour-rot-associated microorganisms.

Isolation and identification sour rot-associated microorganisms. Each sampled berry was placed into a separate $50-\mathrm{ml}$ Falcon tube with $5 \mathrm{ml}$ of distilled water and vortexed for $20 \mathrm{~s}$; then, $100 \mu \mathrm{l}$ of the rinsate was streaked onto plates of both yeast peptone dextrose (YPD) (2\% peptone, $1 \%$ yeast extract, $2 \%$ glucose, and 2\% agar) and yeast peptone mannitol (YPM) $(0.3 \%$ peptone, $0.5 \%$ yeast extract, $2.5 \%$ mannitol, and $1.5 \%$ agar) agar media. The berry was then macerated, and $100 \mu \mathrm{l}$ of the expressed juice was streaked on additional YPD and YPM agar plates. Plates were incubated at $24^{\circ} \mathrm{C}$ for 3 days, or until distinct colonies developed. One colony of each distinct morphological group from each plate was transferred to a new plate of YPD or YPM (i.e., its original culture medium), which was incubated at $24^{\circ} \mathrm{C}$ for a subsequent 3-day period, then stored in a $-4^{\circ} \mathrm{C}$ freezer until subsequent identification.

Using this technique, we recovered 1,246 individual isolates. These were brought out of storage at a single time and separated into 10 different groups based on their morphological characteristics, including color, whole colony shape (punctiform, circular, rhizoid, irregular, or filamentous), edge shape (entire, undulate, lobate, filamentous, or curled), surface texture (smooth, rough, wrinkled, or dry), and elevation (flat, raised, convex, pulvinate, and umbonate). We then determined the origin of each isolate and found that all 10 morphological groups contained isolates recovered from every vineyard site. Each group cumulatively represented organisms associated with 13 to $100 \%$ of all diseased clusters and four of the groups were associated with $\geq 89 \%$ of all diseased clusters. One loop from four arbitrarily chosen plates in each of these four most common groups was transferred to a 50-ml Falcon tube containing $10 \mathrm{ml}$ of sterile distilled water, which was vortexed for $5 \mathrm{~s}$. It was then stored in $\mathrm{a}-4^{\circ} \mathrm{C}$ freezer for subsequent DNA analysis and identification to genus or species level via Sanger sequencing. One of the four isolates from each of these four groups was also chosen arbitrarily for use in subsequent pathogenicity experiments. Four representative isolates from each of the remaining six, less common morphological groups were prepared and stored similarly, and identified via Illumina sequencing.

DNA was extracted by pipetting $400 \mu \mathrm{l}$ of thawed microbial suspension into a test tube containing $5 \mathrm{ml}$ of Tris-EDTA (TE) buffer (10 mM Tris-HCl + 1 mM EDTA, $\mathrm{pH}$ 8.0) plus $0.05 \mathrm{~g}$ of $\mathrm{NaCl}$, which was vortexed for $15 \mathrm{~s}$. Then, $500 \mu \mathrm{l}$ of $10 \%$ sodium dodecyl sulfate was added to the suspension, vortexed for $5 \mathrm{~s}$, and left at room temperature for $15 \mathrm{~min}$. A freeze-thaw sequence consisting of $30 \mathrm{~min}$ in $\mathrm{a}-80^{\circ} \mathrm{C}$ freezer and $5 \mathrm{~min}$ in a $60^{\circ} \mathrm{C}$ water bath was repeated three times to lyse the fungal and bacterial cells, and $750 \mu$ of the solution was transferred to a centrifuge tube along with $750 \mu \mathrm{l}$ of ice-cold isopropanol. The suspension was centrifuged for $10 \mathrm{~min}$ at $9,600 \times g$. The supernatant was carefully discarded from the tube, $500 \mu \mathrm{l}$ of ice-cold $95 \%$ ethanol was added, and the tube was again centrifuged at $9,600 \times g$ for 1 min before discarding the supernatant. The pellet was resuspended in $100 \mu \mathrm{l}$ of TE buffer and this DNA sample was then stored at $4^{\circ} \mathrm{C}$ for subsequent amplification and sequencing.

Two primer sets were used for sequencing all DNA samples. To amplify the V4 domain of bacterial 16s ribosomal RNA (rRNA) genes, primers F515 (5'-GTGTGCCAGCMGCCGCGGTAA-3') and R806 (5'-GGACTACHVGGGTWTCTAAT-3') were used. Fungal internal transcribed spacer (ITS) 1 loci were amplified using primers BITS (5'-CTACCTGCGGARGGATCA-3') and B58S3 (5'-GAGATCCRTTGYTRAAAGTT-3') (Bokulich et al. 2014).

For Sanger sequencing, two polymerase chain reactions (PCR) were performed in 25- $\mu$ l reaction volumes containing GoTaq G2 Green Master Mix (Promega Corporation, Madison, WI), $10 \mathrm{mM}$ each primer, and approximately $10 \mathrm{ng}$ of genomic DNA. Reaction conditions used to amplify the bacterial amplicons consisted of an initial $94^{\circ} \mathrm{C}$ for $3 \mathrm{~min}$; followed by 35 cycles of $94^{\circ} \mathrm{C}$ for $45 \mathrm{~s}, 50^{\circ} \mathrm{C}$ for $60 \mathrm{~s}$, and $72^{\circ} \mathrm{C}$ for $90 \mathrm{~s}$; followed by a final extension of $72^{\circ} \mathrm{C}$ for $10 \mathrm{~min}$ (Bokulich et al. 2014). Reaction conditions used to amplify the fungal amplicons consisted of an initial $95^{\circ} \mathrm{C}$ for 2 min; followed by 40 cycles of $95^{\circ} \mathrm{C}$ for $30 \mathrm{~s}, 55^{\circ} \mathrm{C}$ for $30 \mathrm{~s}$, and $72^{\circ} \mathrm{C}$ for $60 \mathrm{~s}$; and a final extension of $72^{\circ} \mathrm{C}$ for $5 \mathrm{~min}$. (Bokulich et al. 2014). All amplifications were performed in a C1000 Touch Thermal Cycler (Bio-Rad Laboratories Inc., Hercules, CA). To verify amplification of target sequences, PCR products were separated and visualized on a $1.5 \%$ agarose gel (Bio-Rad Laboratories Inc.) stained with ethidium bromide in $1 \times$ Tris-acetate EDTA buffer at $100 \mathrm{~V}$ for $1 \mathrm{~h}$. Photographs of the gel were taken on a KODAK Gel Logic 200 Imaging System (Eastman Kodak Company, Rochester, NY). Amplicons were sequenced at Cornell University's Biotechnology Resource Center in Ithaca, NY.

For Illumina sequencing, Genomic DNA was sent to the Cornell University DNA Sequencing facility in Ithaca, NY for 250-bp paired-end sequencing on the Illumina MiSeq machine. For each sample, two separate runs were performed. Both forward primers were modified to contain a unique 8-bp barcode. Quality filtering, read processing, and operational taxonomic unit (OTU) assignment was conducted in Qiime 1.9.1 (Caporaso et al. 2010b). Sequences were trimmed once there were three consecutive bases with PHRED scores less than 20. Sequences less than 100 nucleotides were discarded. Open and closed reference OTU-picking methods used uclust and a pairwise identity of $97 \%$ (Edgar 2010). Alignment to greengenes 13_5 was done using PyNAST and alignment to UNITE 7_97 was conducted using the BLAST alignment method (Altschul et al. 1990; Caporaso et al. 2010a; DeSantis et al. 2006; Kõljalg et al. 2013). OTU with less than $0.0001 \%$ of the total abundance of the biom file were filtered out. Analysis was done in STAMP v2.1.3 and unclassified reads were not included in the analysis but they were kept to calculate abundance frequencies (Parks et al. 2014).

Single isolates from the two most abundant morphological groups were also submitted to the Fungus Testing Laboratory at the University of Texas in San Antonio to confirm their identification, conducted via combined phenotypic characterization and DNA sequencing of the ITS region and D1/D2 region of the large subunit of the 28S rRNA gene.

Quantification of ethanol and acetic acid. Acetic acid and ethanol concentrations in samples prepared for HPLC analysis were quantified using modifications to the method previously described by Castellari (2001). A 20- $\mu$ l aliquot of each sample was injected onto a Rezex ROA-Organic Acid H+ ion-exclusion column (Phenomenex, Torrance, CA) at $45^{\circ} \mathrm{C}$. Analytes were resolved isocratically using a mobile phase consisting of $6 \%$ ( $\mathrm{vol} / \mathrm{vol})$ acetonitrile and $0.005 \mathrm{~N}$ sulfuric acid in water. Both analytes were 
quantified using external standard curves (run in triplicate, $R^{2}>$ 0.9999 ). The acetic acid was quantified using a photodiode array detector monitoring $210 \mathrm{~nm}$ and the ethanol was quantified using a refractive index detector set in positive polarity mode and thermostated to $45^{\circ} \mathrm{C}$. All samples were analyzed using a Prominence HPLC System (Shimadzu, Kyoto, Japan) with an inline degasser, binary pumps, autoinjector, thermostated column compartment, diode array, and refractive index detectors. Data analysis was performed using LCsolution version 1.25 (Shimadzu).

Inoculations and disease assessment. Supermarketpurchased grape berries of Vitis vinifera 'Red Globe' (17 to 19 Brix) were surface sterilized in a $70 \%$ ethanol solution for $5 \mathrm{~min}$, then rinsed in sterile distilled water prior to inoculation. For each experimental unit, three berries were removed from the clusters above the pedicel to avoid wounding them using surface-sterilized scissors, wounded using a sterile toothpick inserted into the center of the berry and rotated three times, and inoculated by pipetting $50 \mu$ of a microbial suspension into the wound. All three inoculated berries were then placed on a 20 -mm filter paper disc moistened with sterile distilled water in a $137-\mathrm{ml}$ polypropylene specimen container (Fisher Scientific), the lid was fastened, and the treatments were incubated at $24^{\circ} \mathrm{C}$ with photocycles of $12 \mathrm{~h}$ each of light and darkness for 5 or 8 days, depending on the experiment. Unless otherwise noted, there were four replicate experimental units per treatment, and each experiment was repeated.

Following incubation in each appropriate experiment, the development (or not) of sour rot was assessed on the basis of two criteria. The first criterion was a qualitative rating of visual symptoms on a 0 -to- 4 scale, where $0=$ berry is symptomless and completely intact; 1 = berry is completely intact, with some discoloration of the skin only around the wound site; $2=$ berry is entirely intact but with obvious discoloration of the skin extending beyond the wound site; 3 = majority of the berry skin is discolored but berry is still intact; and $4=$ berry is no longer intact, the inner pulp is liquefied and leaking, and the skin is completely discolored (characteristic sour rot symptoms). A single rating was assigned to each three-berry experimental unit. Disease ratings were analyzed through construction of a mosaic plot (Hartigan and Kleiner 1981), a graphical representation of a two-way frequency table showing treatment by disease rating, with the length of each rectangle within a plot proportional to the disease rating frequency. The second criterion was a quantitative measurement of acetic acid, obtained by subsequently macerating each three-berry sample and subjecting the expressed juice to HPLC analysis, as described above. The ethanol concentration of each sample also was determined from this macerate. An inoculation treatment was not considered to have resulted in sour rot unless (i) the mean disease rating was $>3.0$ when averaged across all samples within a treatment, indicating that the berries in at least one sample per treatment had surpassed the discoloration stage and lost integrity; and (ii) the acetic acid concentration was $\geq 0.83 \mathrm{~g} / \mathrm{liter}$, based upon the lowest mean concentration, minus the standard error of the mean, from the 2013 to 2015 field samples as provided below.

In preliminary studies utilizing this inoculation technique with various microbial isolates, we did not obtain typical sour rot symptoms as seen in the field. Therefore, Drosophila flies were included as an additional variable in all subsequent inoculation experiments, based on the ubiquitous association of these insects with sour rot in the field and the previously cited research in which drosophilids were considered to play a role in disease development.

Inclusion of Drosophila melanogaster. A line of colonyraised Drosophila melanogaster was reared for 10 to 14 days on Formula 4-24 Instant Drosophila Medium (Carolina Biological Supply, Burlington, NC) in an incubator at $24^{\circ} \mathrm{C}$ with a photoperiod of $13 \mathrm{~h}$ of light and $11 \mathrm{~h}$ of darkness at 50 to $60 \%$ relative humidity. Flies were released into a 24-by-24-by-24-cm sleeve cage (Bioquip, Rancho Dominguez, CA) that was disinfested with $70 \%$ ethanol prior to use. A plastic aspirator, also disinfested with $70 \%$ ethanol prior to use, was utilized to collect and then release 10 flies at one time into each designated specimen container enclosing the inoculated berries. All specimen containers, regardless of whether or not they contained flies, were placed on a shelf at room temperature with a photoperiod of $13 \mathrm{~h}$ of light and $11 \mathrm{~h}$ of darkness to ensure that the flies remained active.

Preparation of axenic Drosophila spp. Drosophila spp. are known to vector microorganisms on their bodies and in their guts, particularly yeast and AAB (Broderick et al. 2014; Chandler et al. 2011; Koyle et al. 2016; Staubach et al. 2013; Wong et al. 2011). Thus, following our initial inoculation experiments with the lab colony of $D$. melanogaster, where it became clear that these insects were exerting a significant effect, we prepared axenic flies (lacking gut or surface microbiota) for subsequent use following the methods of Koyle et al. (2016), with minor modifications. Approximately 300 colony-reared $D$. melanogaster were released into a sleeve cage containing grape-juice agar plates $(10 \mathrm{~g}$ of torula yeast [number 1720; Bio-Serve, Flemington, NJ], $10 \mathrm{~g}$ of glucose, $1 \mathrm{~g}$ of agar [number 7060; Bio-Serve], $10 \mathrm{~g}$ of supermarket-purchased frozen grape juice concentrate, and $100 \mathrm{ml}$ of water), with a paste containing $1 \mathrm{~g}$ of active dry yeast (Red Star, Milwaukee, WI) in $15 \mathrm{ml}$ of water smeared on the hardened agar. The flies were left to lay eggs overnight in a $24^{\circ} \mathrm{C}$ incubation chamber. Eggs were then collected within a biosafety cabinet by rinsing the agar plate with distilled water and pushing the slurry over a sieve made out of nylon mesh in plastic bushing; then, the bushing was placed in a $120-\mathrm{ml}$ specimen container and the eggs were dechorionated by immersing them in three $90-\mathrm{ml}$ washes of $0.6 \%$ sodium hypochlorite for 2.5 min each followed by three rinses of sterile water. Thirty of these
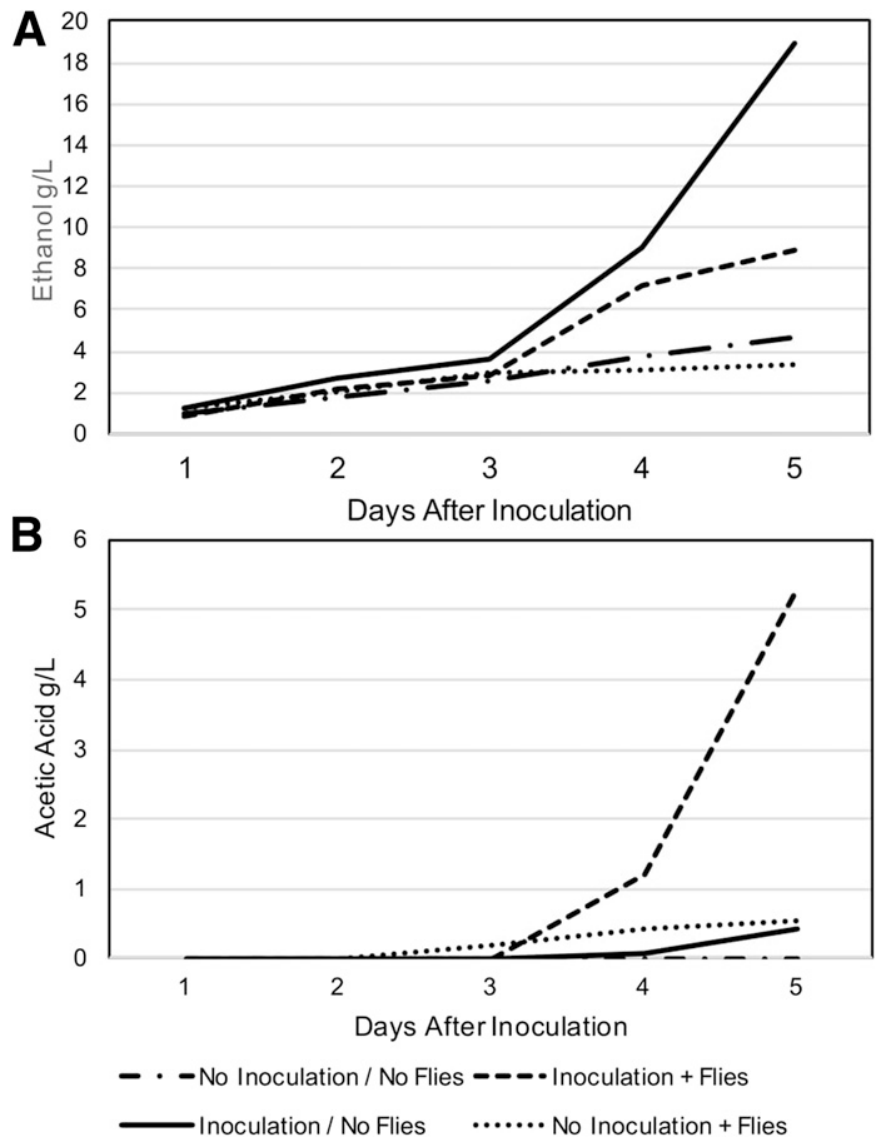

Fig. 1. Mean daily A, ethanol and B, acetic acid accumulation over 5 days in Vitis vinifera 'Red Globe' berries with and without (i) inoculation with a suspension of Saccharomyces cerevisiae plus Acetobacter aceti and (ii) exposure to wild-type Drosophila melanogaster. Each data point represents the mean of six four-berry samples, obtained by combining the data from two runs of the experiment in which there were three replicates per treatment. 
eggs at a time were then transferred, using a surface-sterilized paintbrush, to a $50-\mathrm{ml}$ Falcon tube containing $7.5 \mathrm{ml}$ of sterile yeastglucose diet ( $50 \mathrm{~g}$ of brewer's yeast, $50 \mathrm{~g}$ of glucose, and $6 \mathrm{~g}$ of agar in $500 \mathrm{ml}$ of distilled water, autoclaved at $121^{\circ} \mathrm{C}$ ), and the tubes were placed in an incubator at $24^{\circ} \mathrm{C}$ with a photoperiod of $13 \mathrm{~h}$ of light and $11 \mathrm{~h}$ of darkness. When adults developed, 10 axenic flies and 10 lab colony flies (positive control) were placed separately on each of two plates containing YPD and YPM agar, the lids were affixed, and the plates were incubated at $24^{\circ} \mathrm{C}$ for 2 days. If no microbial colonies developed from them, the axenic flies were then

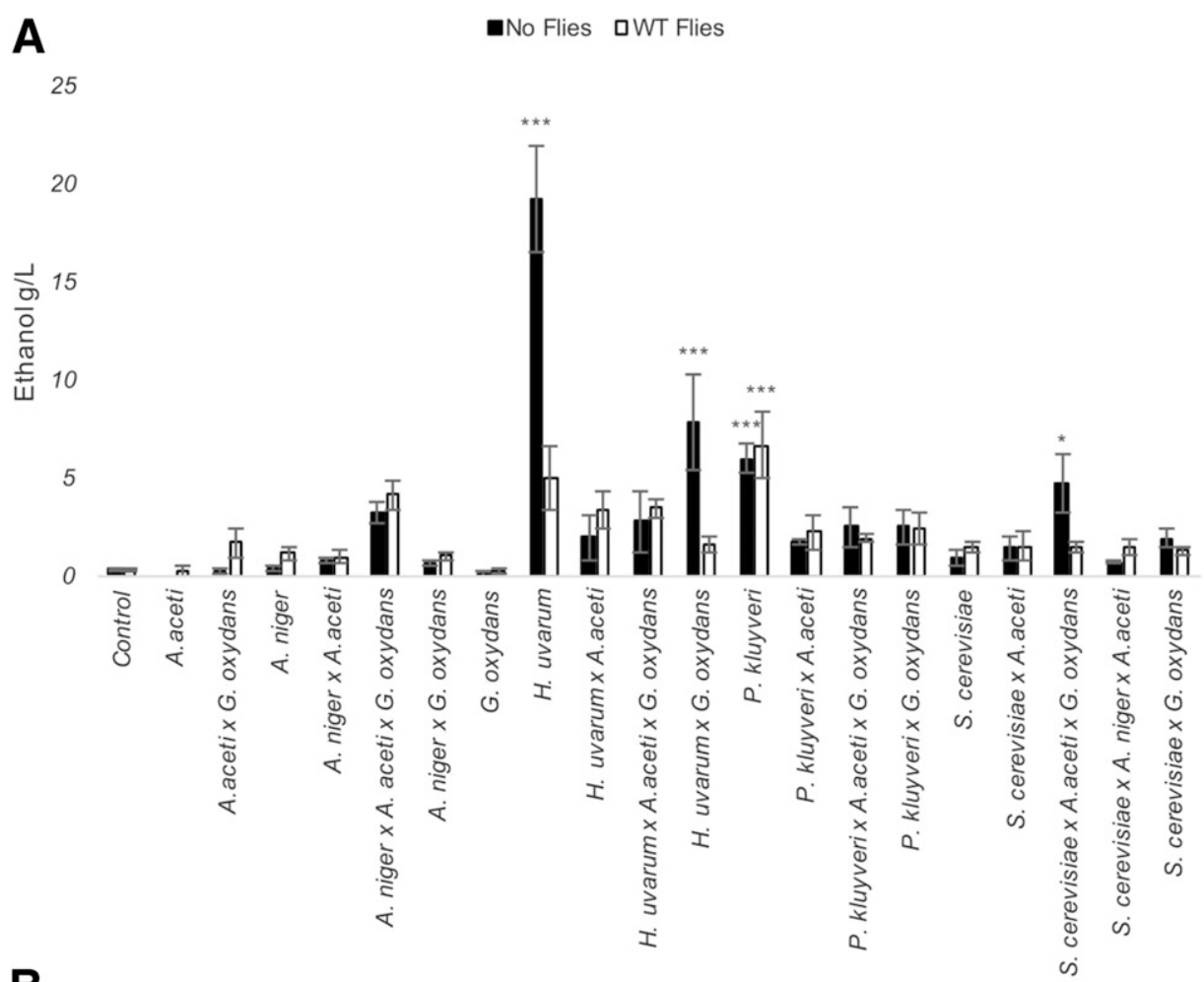

B

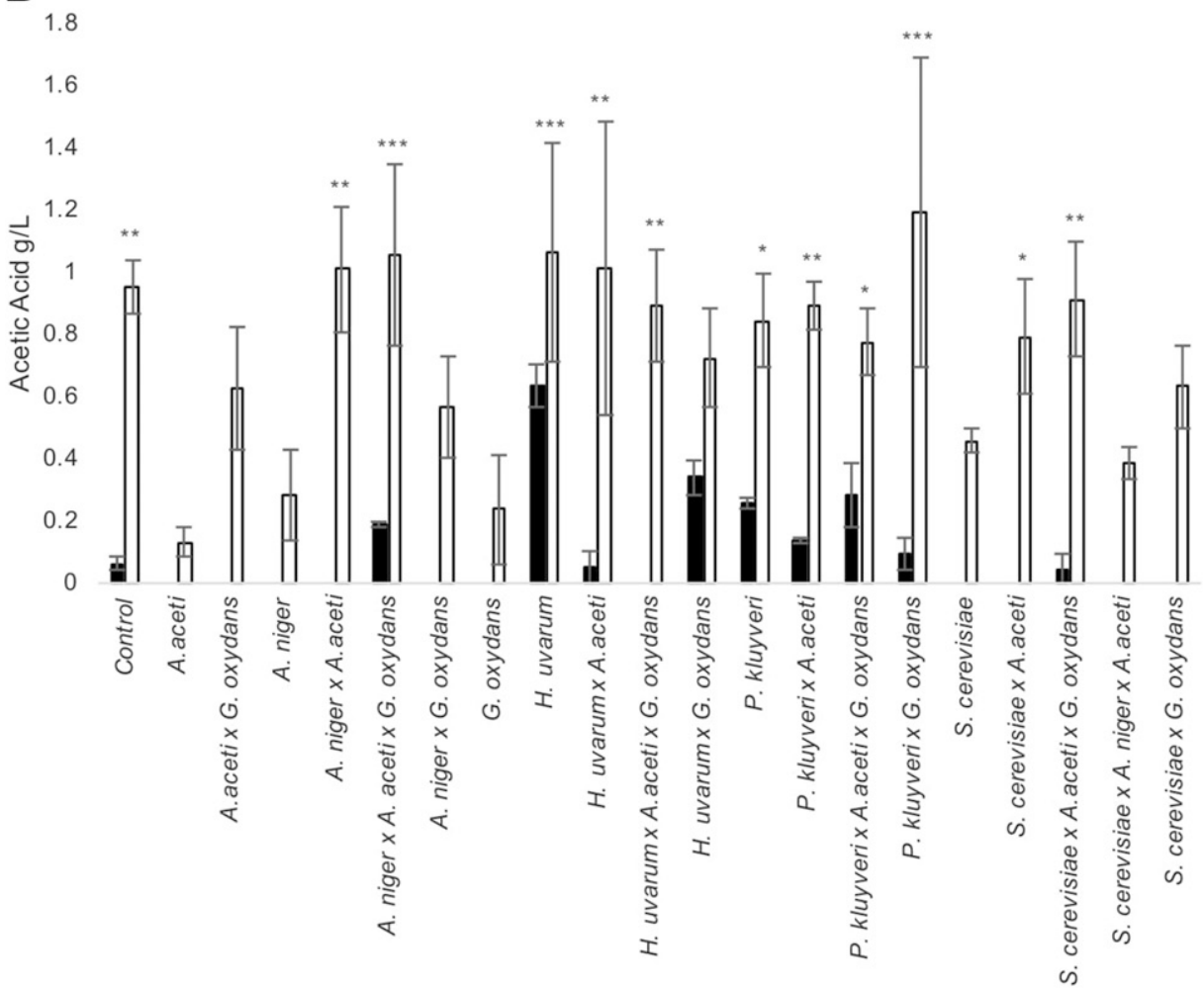

Fig. 2. Mean A, ethanol and B, acetic acid concentration in Vitis vinifera 'Red Globe' grape berries 5 days after inoculation with various isolates from the American Type Culture Collection and combinations thereof, with and without exposure to wild-type (WT) Drosophila melanogaster fruit flies during the incubation period. Expressed values represent the means from eight three-berry samples, obtained by combining the data from two runs of the experiment in which there were four replicates per treatment; error bars represent the standard error of the mean. Asterisks $\left(^{*}\right)$ above a bar denote a statistically significant difference relative to the noninoculated treatment not exposed to $D$. melanogaster, as determined by Dunnett's test: * indicates $P=0.05$, ** indicates $P=0.01$, and *** indicates $P<0.001$. 
utilized in selected inoculation treatments according to the protocol described above for the lab colony flies.

Evolution of ethanol and acetic acid. In a 2-by-2 factorial design, berries of $V$. vinifera 'Red Globe' were (i) either inoculated with a combination of Saccharomyces cerevisiae and Acetobacter aceti (isolates obtained from the American Type Culture Collection [ATCC]) or not and (ii) either exposed to lab colony flies or not, using the procedures described previously. At the end of each of the five subsequent days following inoculation, all berries from each designated specimen cup were macerated and the expressed juice was transferred to a 2-ml tube and frozen for subsequent HPLC analysis for acetic acid and ethanol concentration. There were three replicate cups for each treatment-timing combination, each containing four berries. We conducted two runs of this experiment.

Pathogenicity experiments. Inoculation with ATCC cultures. Individual isolates of species of filamentous fungi, yeast, and bacteria reported previously to be associated with sour rot were obtained from the ATCC. To produce inoculum, S. cerevisiae (ATCC 204508), Pichia kluyveri (ATCC 24209), and Hanseniospora uvarum (ATCC 32369) were cultured on YPD; A. aceti (ATCC 15973) and Gluconobacter oxydans (ATCC 33448) on YPM; and Aspergillus niger (ATCC 16888) on potato dextrose agar. All isolates were incubated at $24^{\circ} \mathrm{C}$ for 3 to 7 days. Then, one colony from each isolate was transferred to a 50-ml Falcon tube containing $5 \mathrm{ml}$ of sterile distilled water, which was vortexed for $5 \mathrm{~s}$; the spore concentration of every suspension was determined with a hemacytometer and adjusted to $9.0 \times 10^{6}$ cells $/ \mathrm{ml}$ with sterile distilled water; and inoculations were performed according to the method detailed previously. In the first set of experiments, inoculation treatments were either exposed to $10 \mathrm{D}$. melanogaster adults from the lab colony or not exposed to flies. After 5 days of incubation, each three-berry sample was rated for sour rot symptoms on the 0 -to-4 scale and prepared for ethanol and acetic acid analysis, as described previously. In a second experiment, berries were inoculated with the same isolates and combinations thereof using the same procedures, except (i) the inoculated berries were either exposed to 10 axenic (rather than wild-type) D. melanogaster adults or not exposed to flies and (ii) the treatments were evaluated after 8 days of incubation rather than 5. Both experiments were repeated once.

Inoculation with field-recovered isolates. The representative isolates from each of the four most commonly recovered morphological groups (i.e., those isolated from $\geq 89 \%$ of all symptomatic field samples) were tested for pathogenicity, both individually and in various combinations, according to the procedures described above. All inoculation treatments were either exposed to 10 axenic D. melanogaster adults or not exposed to flies. Disease symptoms were assessed using the previously described 0-to-4 scale and ethanol or acetic acid concentrations were evaluated after 8 days of incubation. This experiment was repeated once.

Microbial contribution of lab-colony D. melanogaster. To investigate the potential development of sour rot resulting from transfer of their resident microbiota to grape berries, 10 live wildtype $D$. melanogaster adults from the lab colony were released onto plates of YPD medium, which were incubated for 3 days at $24^{\circ} \mathrm{C}$ at a photoperiod of $13 \mathrm{~h}$ of light and $11 \mathrm{~h}$ of darkness. Then, distilled water was poured onto each plate to create a slurry, and either (i) the resulting suspension was pipetted into a $1.5-\mathrm{ml}$ collection tube and used to inoculate grape berries as described above or (ii) a $400-\mu l$ aliquot was transferred to a separate $1.5-\mathrm{ml}$ collection tube for DNA extraction and Illumina sequencing, as described previously. Inoculated and noninoculated berries were exposed to either axenic flies or no flies; a third group, of noninoculated berries, was exposed to wild-type lab-colony flies. Disease symptoms were assessed using the previously described 0 -to- 4 scale and ethanol or acetic acid concentrations were evaluated after 8 days of incubation. This experiment was repeated once.
Botrytis rot-sour rot interaction. To investigate the potential interaction between the development of Botrytis bunch rot and sour rot on grape clusters, we collected (i) apparently healthy, symptomless clusters; (ii) Botrytis rot clusters; and (iii) sour rotaffected clusters from two commercial vineyards of $V$. vinifera 'Riesling' in southern Tasmania, Australia and one research vineyard of Vitis interspecific hybrid cultivar Vignoles in Geneva, NY. All symptomatic clusters were visually determined to have approximately $50 \%$ disease severity of either Botrytis bunch rot or sour rot but appeared to be free of the other disease. Clusters were cut from the vine using pruning shears and bagged individually in low-density polyethylene bags. In independent experiments examining these two groups, five clusters per experimental unit were arranged on a flat surface in the laboratory within a 3.8-liter sealable plastic bag: four in a square, with their edges $1 \mathrm{~cm}$ apart, and one in the center of this square, with its edges $1 \mathrm{~cm}$ from the edge of each surrounding cluster. There were four replicate bags per treatment. The treatments consisted of four Botrytis rot clusters surrounding one sour rot-affected cluster; four Botrytis rot clusters

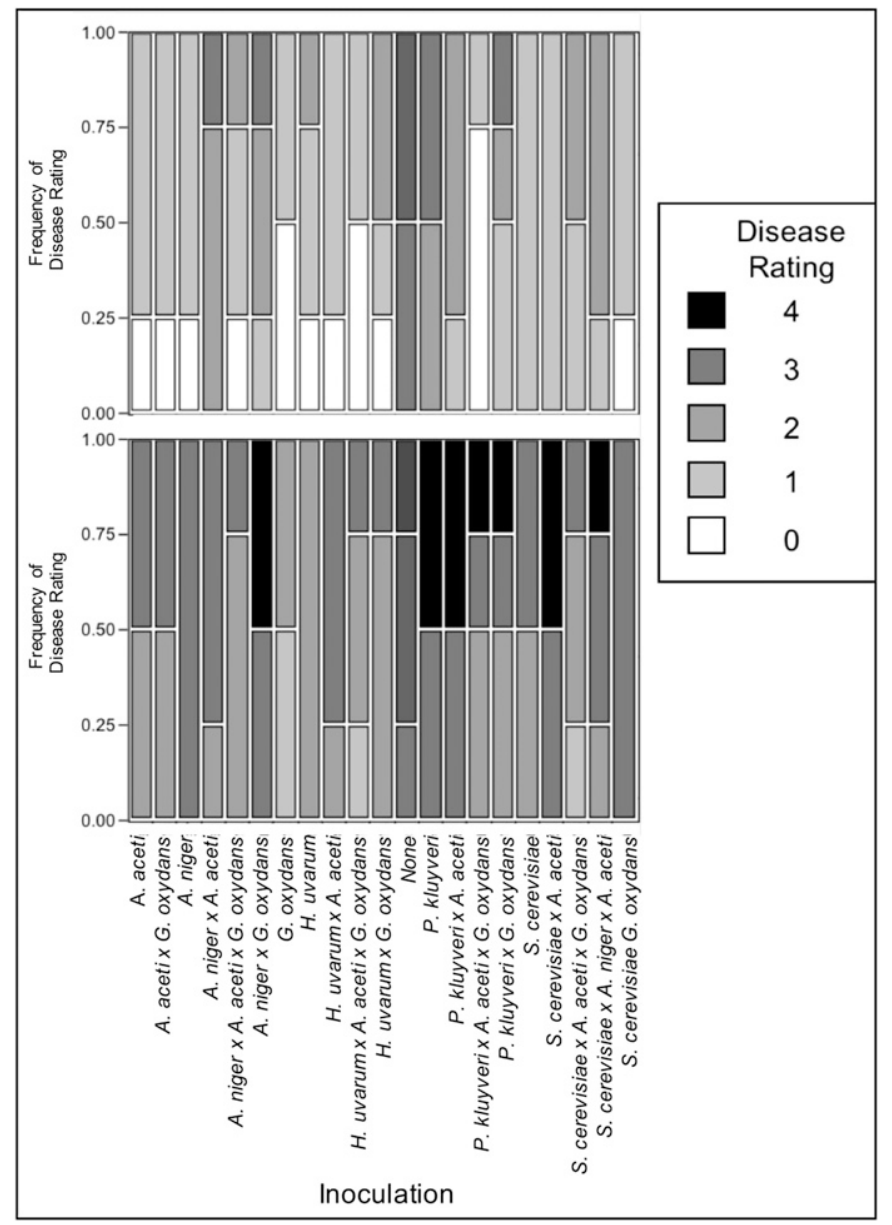

Fig. 3. Frequency of disease severity on individual Vitis vinifera 'Red Globe' grape berries inoculated with isolates of six fungal or bacterial species obtained from the American Type Culture Collection, either alone or in various combinations, and either not exposed (top) or exposed (bottom) to wild-type Drosophila melanogaster fruit flies during a 5-day incubation period. Frequencies presented are for eight three-berry samples and represent the combined data from two independent runs of the experiment, in which there were four replicate experimental units per treatment. Disease severity is based on a 0 -to- 4 scale, where $0=$ berry is symptomless and completely intact; $1=$ berry is completely intact, with some discoloration of the skin only around the wound site; 2 = berry is entirely intact but with obvious discoloration of the skin extending beyond the wound site; $3=$ majority of the berry skin is discolored but berry is still intact; and $4=$ berry is no longer intact, the inner pulp is liquefied and leaking, and the skin is completely discolored (characteristic sour rot symptoms). 
surrounding one healthy cluster; four healthy clusters surrounding one sour rot-affected cluster; and a control containing five healthy clusters. All treatments were incubated for 10 days at room temperature with a photoperiod of $12 \mathrm{~h}$ of light and $12 \mathrm{~h}$ of darkness, at the end of which all clusters were visually rated for the severity of each disease (percent cluster area symptomatic). The presence of sporulation of $B$. cinerea and the visual loss of berry integrity accompanied by the smell of acetic acid characteristic of sour rot were used as the basis for assessing and differentiating between the two diseases.

A - No Flies aAxenic Flies

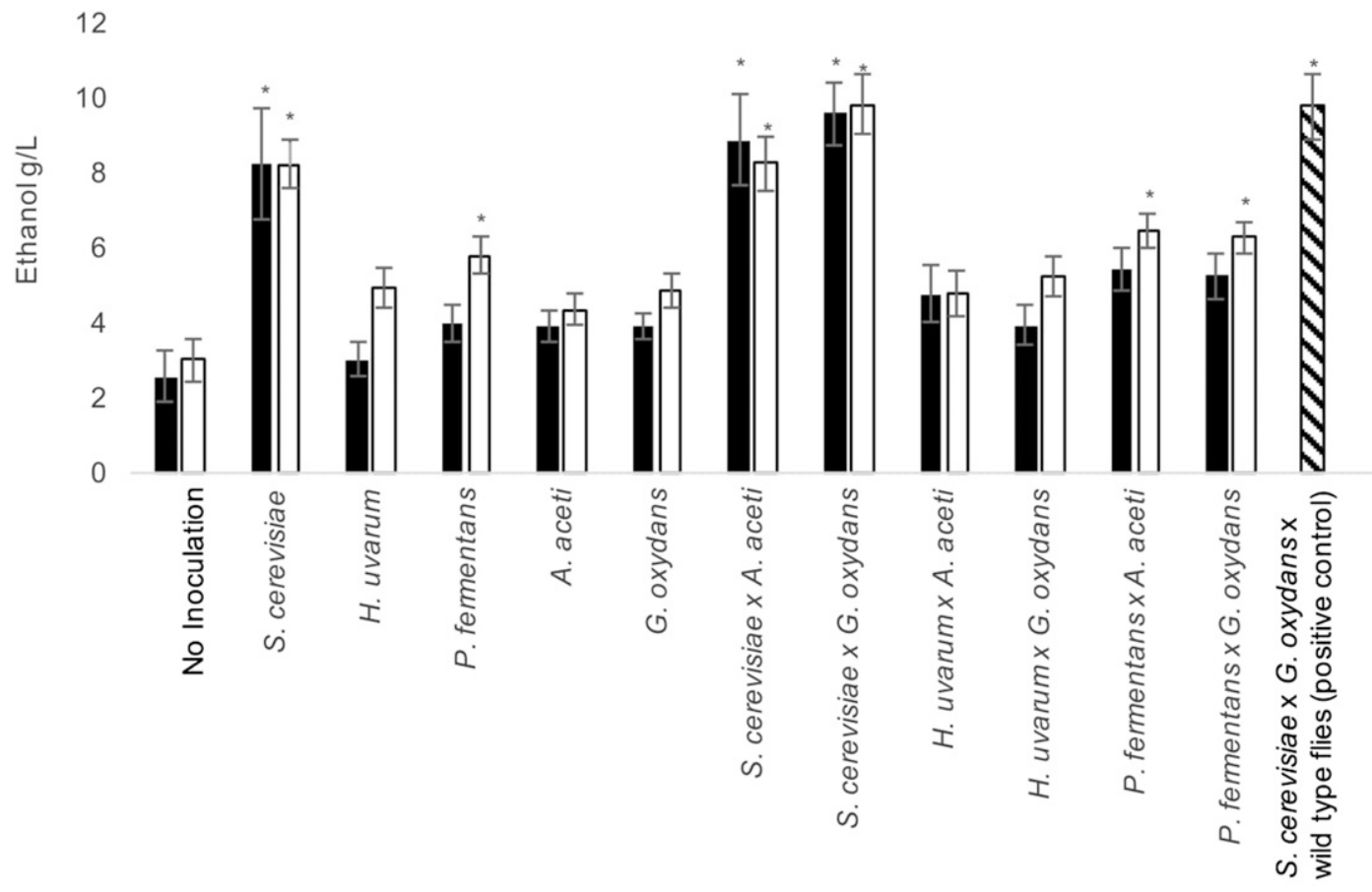

B

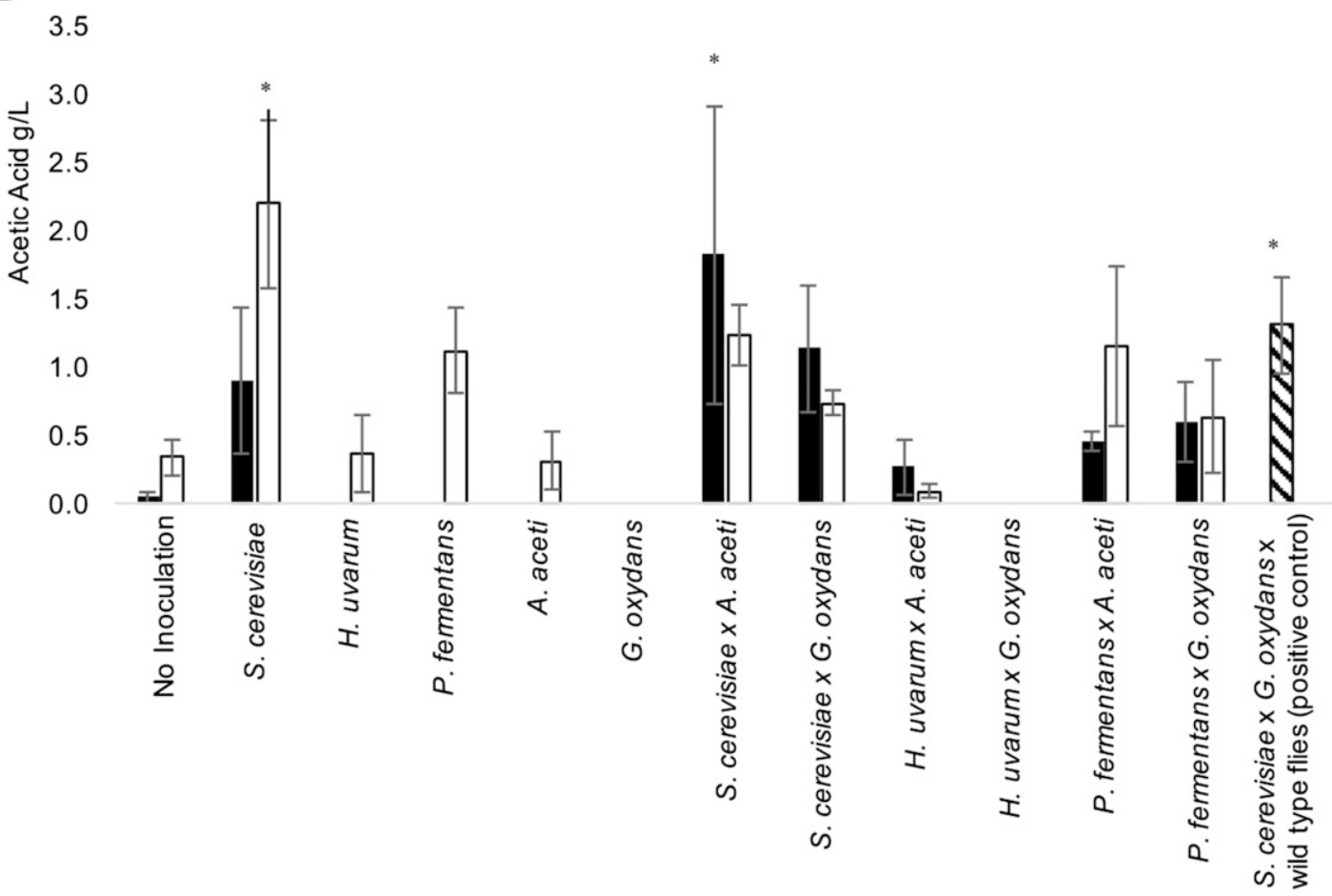

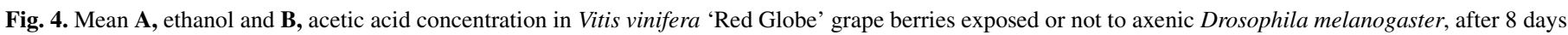

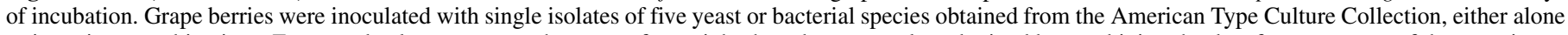

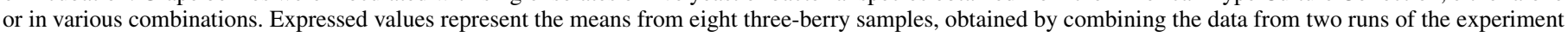

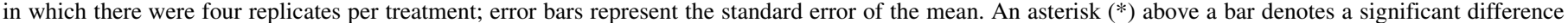
$(P=0.05)$ relative to noninoculated berries not exposed to axenic D. melanogaster, as determined by Dunnett's test. 
Data analysis. All analyses were conducted using the JMP Pro v. 11 statistical software. For analysis of ethanol and acetic acid concentration in field samples, measurements from all samples of a particular year were used to calculate the mean and standard error of the mean (SEM) of the sample set. For analysis of ethanol and acetic acid concentrations in the daily evolution experiment, both repetitions of the experiment were combined into a single data set after determining homogeneity of variance via Levene's test and analyzed for the mean and SEM of each treatment. The effect of Drosophila inclusion (+ or -) on the mean ethanol and acetic acid concentrations of inoculated berries was compared each day using two-sided $t$ tests. For analysis of ethanol and acetic acid in pathogenicity experiments, samples from both repetitions were combined into one data set after determining their homogeneity of variance via Levene's test to determine the mean and SEM of each treatment. The effect of Drosophila inclusion (+ or -) on the mean ethanol and acetic acid concentrations for each individual treatment at the end of the experiment was determined using a two-sided $t$ test, and all treatments were compared with the untreated control using Dunnett's test. Disease ratings from both repetitions of each experiment were also combined into one data set, which was used to determine the frequency of each rating and the mean rating for each treatment. For analysis of the Botrytis rot-sour rot experiments, both repetitions were combined into one data set after confirming homogeneity of variance via Levene's test and then analyzed to determine the mean and SEM for each treatment.

\section{RESULTS}

Ethanol and acetic acid concentration in field samples. The mean $( \pm$ SEM $)$ for the ethanol and acetic acid concentrations of the whole-cluster samples in 2013 were $2.65 \pm 0.42$ and $2.41 \pm 0.37$ g/liter, respectively. In 2014 and 2015, when samples were confined to three symptomatic berries from predetermined regions of the cluster, the mean values for ethanol were $1.12 \pm 0.096$ and $1.16 \pm$ $0.16 \mathrm{~g} /$ liter, respectively; those for acetic acid were $0.95 \pm 0.12$ and $2.20 \pm 0.30 \mathrm{~g} / \mathrm{liter}$, respectively. Because we considered the latter measurements to be more precise than the whole-cluster measurements of 2013, as confirmed by their lower standard errors, we established the quantitative acetic acid threshold of $0.83 \mathrm{~g} / \mathrm{liter}$ as a criterion for defining the development of sour rot in our inoculation experiments, as noted previously. This was determined by taking the mean of the 2014 field samples (i.e., the lower of the 2 years) minus the standard error of that sample set.

Accumulation of ethanol and acetic acid. The mean ethanol level measured across all treatments was $1.1 \mathrm{~g} /$ liter on the first day following inoculation (Fig. 1). This concentration had tripled by 3 days after inoculation (DAI) when inoculated berries were not exposed to flies, increasing to 8- and 16-fold times that on the first day at 4 and 5 DAI, respectively. Ethanol accumulated similarly over the first 3 days when inoculated berries were exposed to flies but the rate of accumulation slowed thereafter, with the concentration by $5 \mathrm{DAI}$ only half as great as for the inoculated berries not exposed to flies. Trace level of acetic acid were detected at $3 \mathrm{DAI}$, and the concentration increased dramatically over the next 2 days in inoculated berries exposed to flies (Fig. 1). In contrast, little acetic acid developed within the inoculated berries that were not exposed to flies, with the final concentration at 5 DAI only approximately $10 \%$ of that for similar berries exposed to the flies (Fig. 1). This acetic acid evolution in the fly-exposed berries, which was most pronounced during the final day of incubation, was coincident with a divergence of ethanol accumulation in the two inoculated treatments (i.e., the ethanol concentrations were not significantly different between the two when assayed at 4 DAI whereas the concentration was more than twice as high at 5 DAI in berries not exposed to flies $[P=0.0248]$ ). The ethanol concentration within noninoculated berries was measurable but relatively low, both with and without exposure to D. melanogaster; however, acetic acid developed within noninoculated berries only when they also were exposed to the flies, at a final concentration approximately $10 \%$ of that in the inoculated berries similarly exposed to flies.

Endophytic microbes. Because we consistently detected ethanol (and, sporadically, acetic acid) in the above and subsequent experiments when wounded berries were not exposed to a source of microbes and standard sterile techniques had been employed, we investigated the possibility that microorganisms responsible for their production are commonly present as endophytes within healthy berries. To do so, we collected berries from healthy, supermarket-purchased Red Globe clusters and symptomless clusters of other cultivars representing $V$. vinifera and Vitis interspecific hybrids, obtained from six vineyards in New York, Washington, and Tasmania, Australia. From each sample, three berries from each of three clusters were surface disinfested for $5 \mathrm{~min}$ in $70 \%$ ethanol and cut in half using a sterile razor blade, and the cut surface of each half was placed on either YPD or YPM agar and incubated for 7 days at $24^{\circ} \mathrm{C}$. The techniques used for subsequent purification and identification of the resulting microbial colonies via Illumina DNA sequencing were as described previously. Various yeast genera, primarily Metschnikowia and Pichia, were consistently isolated from berries at every sample location, as were

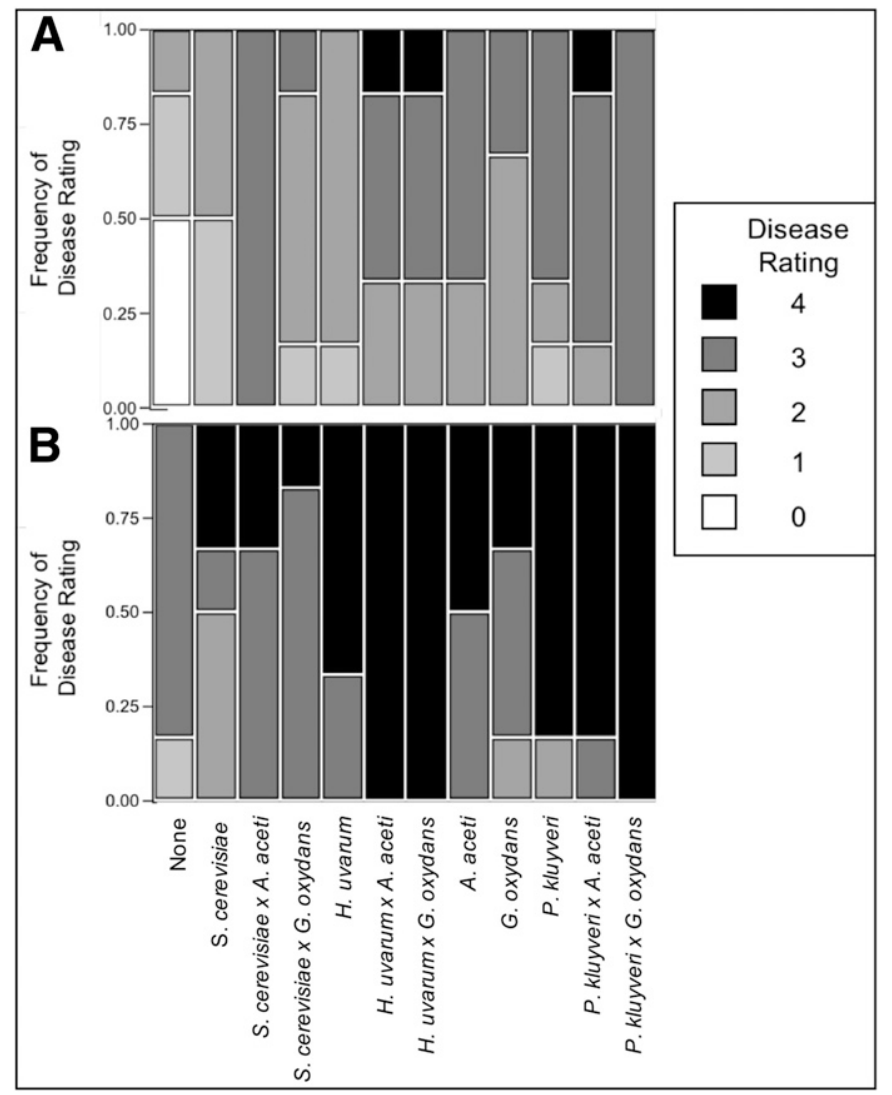

Fig. 5. Frequency of disease severity on individual Vitis vinifera 'Red Globe' grape berries inoculated with isolates of six fungal or bacterial species obtained from the American Type Culture Collection, either alone or in various combinations, and either A, not exposed or B, exposed to axenic Drosophila melanogaster fruit flies during an 8-day incubation period. Frequencies presented are for eight three-berry samples and represent the combined data from two independent runs of the experiment, in which there were four replicate experimental units per treatment. Disease severity is based on a 0-to-4 scale where $0=$ berry is symptomless and completely intact; $1=$ berry is completely intact, with some discoloration of the skin only around the wound site; $2=$ berry is entirely intact, but with obvious discoloration of the skin extending beyond the wound site; 3 = majority of the berry skin is discolored but berry is still intact; and $4=$ berry is no longer intact, the inner pulp is liquefied and leaking, and the skin is completely discolored (characteristic sour rot symptoms). 
bacteria in the genera Bacillus, Pseudomonas, Streptococcus, Acetobacter, and Gluconobacter. Additional details on these methods and results are provided elsewhere (Hall et al. 2019).

Identification of microbes from field samples. Two of the four most common morphologically distinct groups yielded two 251-bp bacterial amplicons whereas the other two yielded two 86-bp fungal amplicons. A BLAST analysis of the bacterial fragments found one to have $98 \%$ (212 of 216) identity with G. cerinus and the second to have $97 \%$ sequence similarity to many members of the family Enterobacteriaceae, primarily Rahnella sp., Yersinia ruckeri,

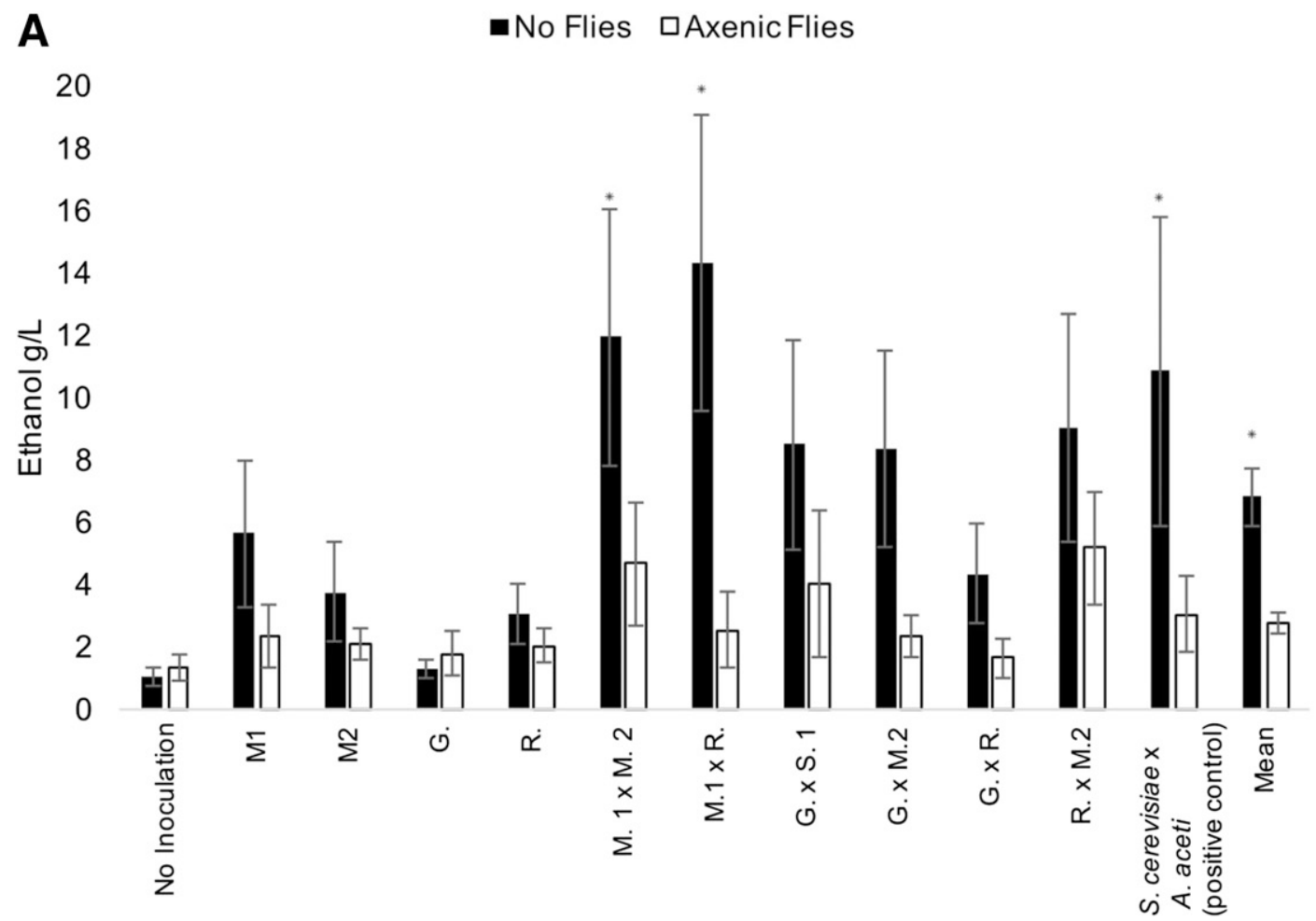

B

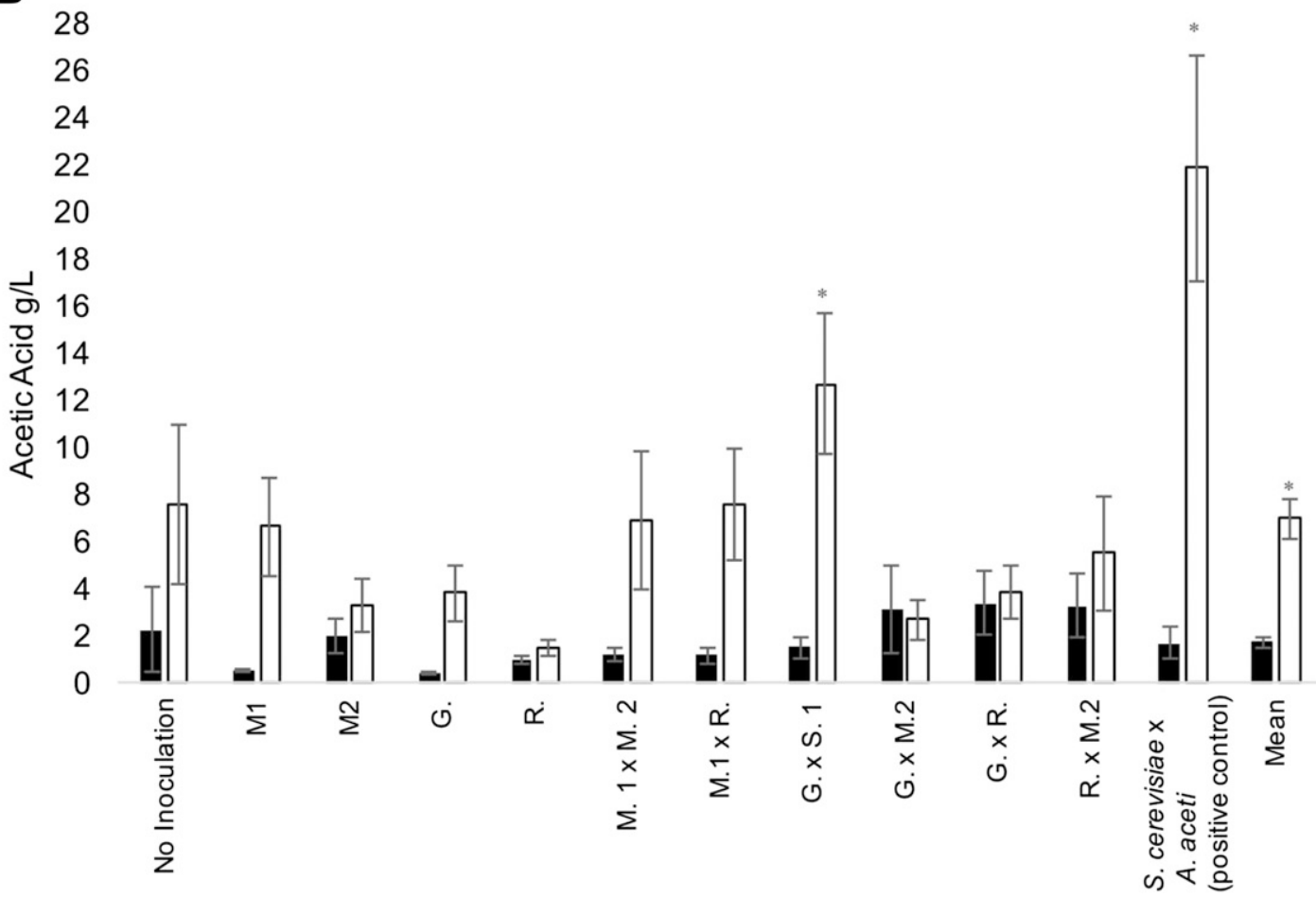

Fig. 6. Mean A, ethanol and B, acetic acid concentration in inoculated Vitis vinifera 'Red Globe' grape berries exposed, or not, to axenic Drosophila melanogaster fruit flies. Grape berries were inoculated with isolates of four yeast and bacterial species recovered from sour rot-affected berries in the Finger Lakes American Viticultural Area, either alone or in various combinations. Isolates were identified as two species of Metschnikowia (M1 and M2), Gluconobacter cerinus (G), and a Rahnella sp. (R). Expressed values represent the means from eight three-berry samples, obtained by combining the data from two runs of the experiment in which there were four replicates per treatment; error bars represent the standard error of the mean. An asterisk $(*)$ above a bar denotes a significant difference $(P=0.05)$ relative to noninoculated berries not exposed to axenic D. melanogaster, as determined by Dunnett's test. 
and Hafnia sp., with the best match being Rahnella sp. UIWRF0013 (accession KR189951.1). The fungal fragments had a 95\% (54 of 57) identity with several yeast species, including Saccharomyces spp. Further sequencing and phenotypic characterization of the two fungal isolates showed that both were members of the genus Metschnikowia.

Of the six remaining morphological groups examined, the one represented by an isolate identified as Acetobacter pasteurianis was

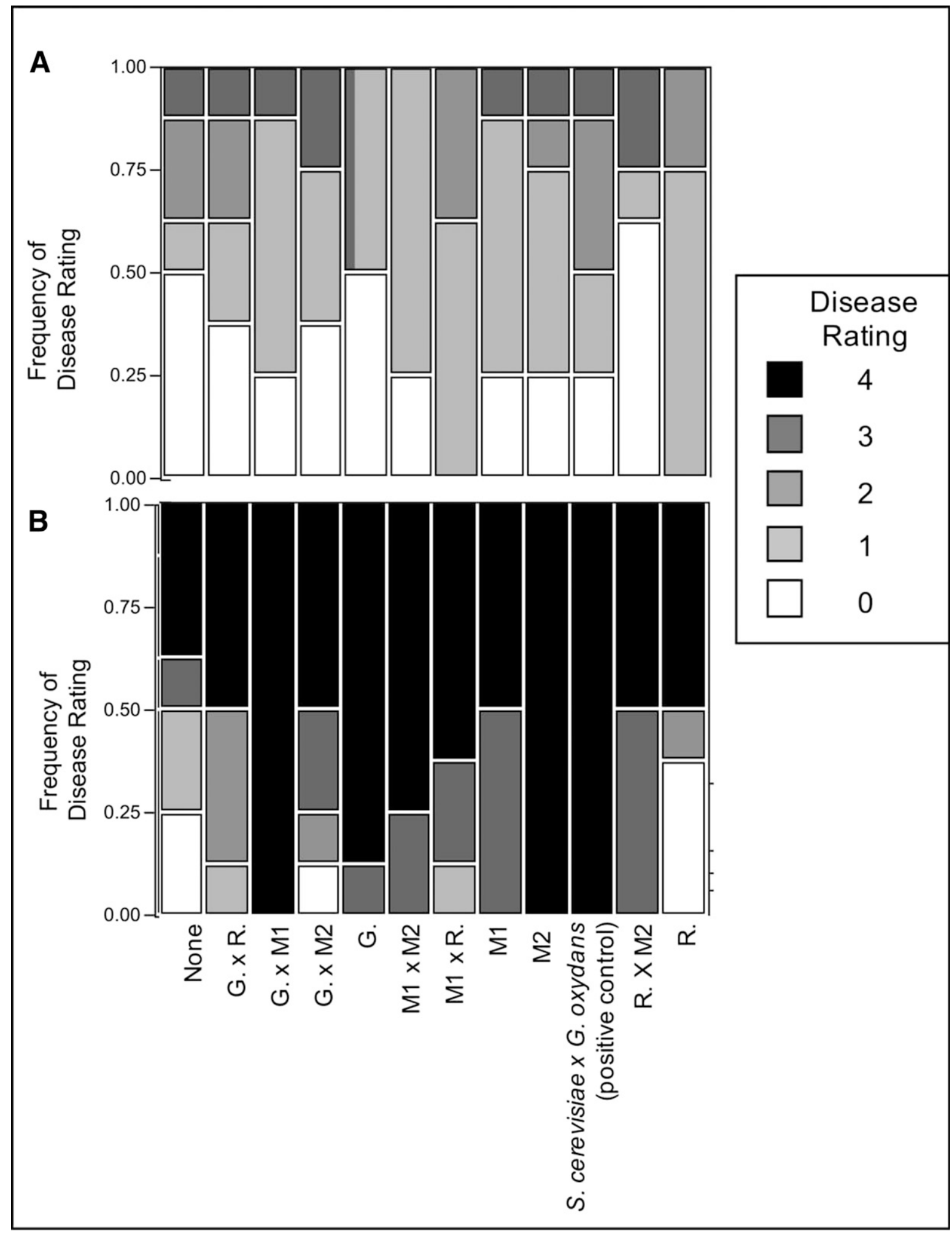

Fig. 7. Frequency of disease severity on individual Vitis vinifera 'Red Globe' grape berries inoculated with isolates of four yeast and bacterial species recovered from sour rot-affected grape berries in the Finger Lakes American Viticultural Area, alone and in various combinations, and either A, not exposed or B, exposed to axenic Drosophila melanogaster fruit flies during a 8-day incubation period. Isolates were identified as two species of Metschnikowia (M1 and M2), Gluconobacter cerinus $(\mathrm{G})$, and a Rahnella $\mathrm{sp}$. (R). Frequencies presented are for eight three-berry samples and represent the combined data from two independent runs of the experiment, in which there were four replicate experimental units per treatment. Disease severity is based on a 0 -to- 4 scale, where $0=$ berry is symptomless and completely intact; 1 = berry is completely intact, with some discoloration of the skin only around the wound site; $2=$ berry is entirely intact but with obvious discoloration of the skin extending beyond the wound site; $3=$ majority of the berry skin is discolored but berry is still intact; and $4=$ berry is no longer intact, the inner pulp is liquefied and leaking, and the skin is completely discolored (characteristic sour rot symptoms). 
recovered from 57\% of the diseased clusters in 2014 but only $8.3 \%$ in 2015 , and that represented by an isolate identified as P. occidentalis was found in $68 \%$ of the diseased clusters in 2014 and $22 \%$ in 2015 . Isolates of the remaining less-common groups (and the frequencies of recovery for the groups that they represented) were identified through BLAST searches of the Illumina sequencing data as members of P. membranifaciens (33.3\% in 2014 and $48.1 \%$ in 2015), P. fermentans (47.6\% in 2014 and $22.2 \%$ in 2015), and Candida inconspicua (14.3\% in 2014 and $26.9 \%$ in 2015 ) or as uncultured bacteria (19\% in 2014 and $18.5 \%$ in 2015).

Pathogenicity experiments. Inoculation with ATCC cultures. In experiments utilizing wild-type $D$. melanogaster, exposure to these insects during the incubation period significantly increased the accumulation of acetic acid in the inoculated berries $(P<$ 0.0001 ) (Fig. 2B). In contrast, little to no acetic acid accumulated in most treatments incubated without exposure to the flies, and the concentration in these treatments never exceeded the $0.83 \mathrm{~g} /$ liter threshold established as a criterion for sour rot development in our study (Fig. 2B). Ethanol accumulations were typically below 2.0 $\mathrm{g} /$ liter and exceeded $4.5 \mathrm{~g} /$ liter in only four sets of inoculations that included the yeast species $H$. uvarum, $P$. kluyveri, or $S$. cerevisiae (Fig. 2A). In three of these four instances, the ethanol concentration was demonstrably higher when inoculated berries were not exposed to D. melanogaster than when they were; whereas, in all four, the converse was true for acetic acid concentration. Only those inoculated berries also exposed to D. melanogaster developed visual symptoms classified as sour rot (i.e., a mean disease rating $>3.0$ ) (Fig. 3). Of those satisfying both the acetic acid and visual criteria,

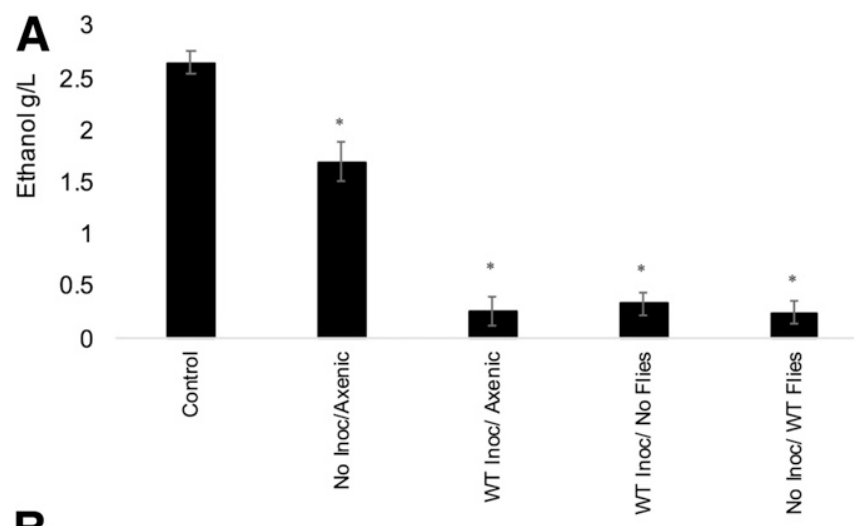

B

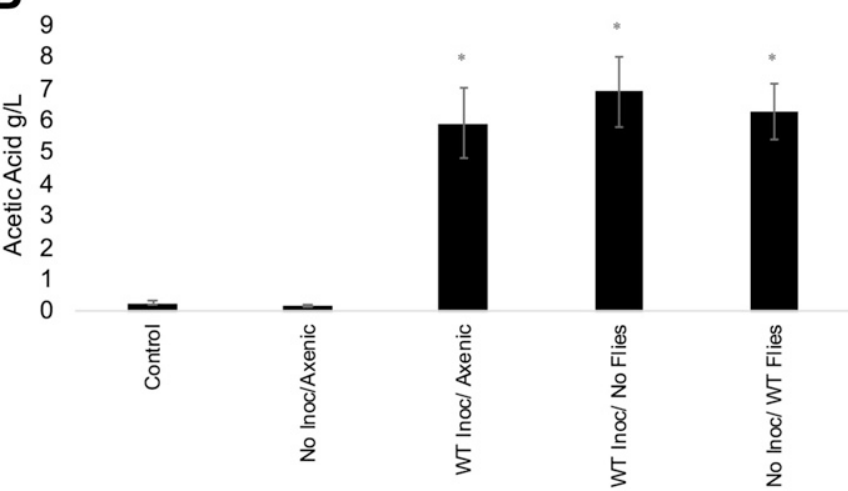

Fig. 8. Mean A, ethanol and $\mathbf{B}$, acetic acid concentration in inoculated Vitis vinifera 'Red Globe' grape berries exposed to either axenic or wild-type (WT) Drosophila melanogaster. Grape berries were inoculated with a suspension of organisms previously recovered from a WT colony of $D$. melanogaster fruit flies, the majority of which were in the orders Enterobacteriales and Saccharomycetales. Expressed values represent the means from eight three-berry samples, obtained by combining the data from two runs of the experiment in which there were four replicates per treatment; error bars represent the standard error of the mean. An asterisk $(*)$ above a bar denotes a significant difference $(P=0.05)$ relative to the noninoculated control treatment, which was not exposed to D. melanogaster flies, as determined by Dunnett's test. only three inoculation treatments, all in combination with exposure to D. melanogaster, caused sour rot symptoms: P. kluyveri $\times$ G. oxydans, $S$. cerevisiae $\times A$. aceti, and $S$. cerevisiae $\times$ G. oxydans.

When berries were inoculated with these same individual organisms and combinations thereof in subsequent experiments and exposed (or not) to axenic D. melanogaster, exposure to the flies affected the accumulation of both ethanol, which was lower in the fly-exposed treatments $(P=0.0482)$, and acetic acid, which was higher $(P=0.0686)$ (Fig. 4$)$. Seven treatments had a mean disease rating $>3.0$, six of which included exposure to axenic flies (Fig. 5). The only inoculation treatments that met both diagnostic criteria for causing sour rot were $S$. cerevisiae $\times A$. aceti, $S$. cerevisiae $\times$ G. oxydans, P. kluyveri $\times$ A. aceti, and P. kluyveri $\times$ G. oxydans, and only with concomitant exposure to axenic flies. The concentration of acetic acid exceeded $0.83 \mathrm{~g} /$ liter in the absence of flies when berries were inoculated with $S$. cerevisiae, alone or in combination with either A. aceti or G. oxydans; however, the mean disease rating in these treatments was $<3.0$ (Fig. 5).

Inoculation with isolates recovered from field samples. When averaged across all inoculation treatments, including the noninoculated control, exposure to axenic D. melanogaster significantly increased the acetic acid concentration relative to treatments not exposed to flies, to 6.98 versus $1.72 \mathrm{~g} /$ liter, respectively, after 8 days of incubation $(P<0.0001)$ (Fig. 6). In contrast, the mean ethanol concentration at the end of the 8-day incubation period decreased with exposure to axenic D. melanogaster, from 6.81 to $2.75 \mathrm{~g} /$ liter $(P<0.0001)$, without and with inclusion of the flies, respectively (Fig. 6). Only those treatments that included flies had a combination of acetic acid levels $\geq 0.83 \mathrm{~g} /$ liter and mean disease ratings $>3.0$ when averaged across all samples within a treatment, and all such treatments except the Rahnella sp. alone produced visual symptoms of sour rot (Fig. 7). All treatments in which either yeast was included or in which Gluconobacter sp. was the only source of inoculum were successful in causing visual symptoms when flies were included.

Macerate obtained from each sample at the end of the experiment was streaked on plates of YPD and YPM. After 4 days of incubation at $24^{\circ} \mathrm{C}$, DNA was recovered from two replicates of each morphologically distinct colony that developed and subjected to Sanger sequencing, using the methods described previously. Identifications from these data showed that we successfully reisolated each of the organisms included in the specific inoculations, in addition to species of Pichia, Candida, and Acetobacter, genera that we determined previously to be common endophytes in grape berries.

Microbial contribution of wild-type D. melanogaster. Sequencing results of the composite inoculum produced from plates exposed to the wild-type fly colony showed that $80 \%$ of the bacterial OTU were from the order Enterobacteriales, 5.3\% from Bacillales, 3.2\% from Burkholderiales, and approximately $1 \%$ each from the orders Actinomycetales, Aeromondales, Lactobacillales, Pseudomonales, and Rhizobiales and superdivision Streptophyta. Nearly all (98.5\%) of the fungal OTU were from the order Saccharomycetales, the majority of which were Metschnikowia spp., with the remaining $1.5 \%$ from Tremellales.

When this composite inoculum was introduced into grape berries, significantly more ethanol developed in the noninoculated control treatment, both with and without exposure to axenic flies, than in the inoculated treatments exposed to axenic flies or in the noninoculated berries exposed to wild-type flies, all of which generated an acetic acid concentration $>5.9$ g/liter (Fig. 8). Significant acetic acid concentrations developed in berries inoculated with the microbes isolated from D. melanogaster, with or without exposure to axenic flies (Fig. 8). However, significant visual symptoms did not develop in berries not exposed to these flies, whereas the vast majority of berries that were exposed developed severe disease symptoms. Noninoculated berries exposed to wild-type flies generated significant acetic acid but had a mean disease rating of 
only 2.0 (Fig. 9). Wounded grape berries that were not inoculated had relatively low concentrations of acetic acid, either with or without exposure to axenic flies (mean of 0.27 and $0.17 \mathrm{~g} / \mathrm{liter}$, respectively) (Fig. 8), and did not develop symptoms consistent with sour rot (mean disease ratings of 0.5 and 1.5 , respectively) (Fig. 9).

Botrytis rot-sour rot interaction. At the end of the 10-day incubation period, all treatments in which sour rot had developed on
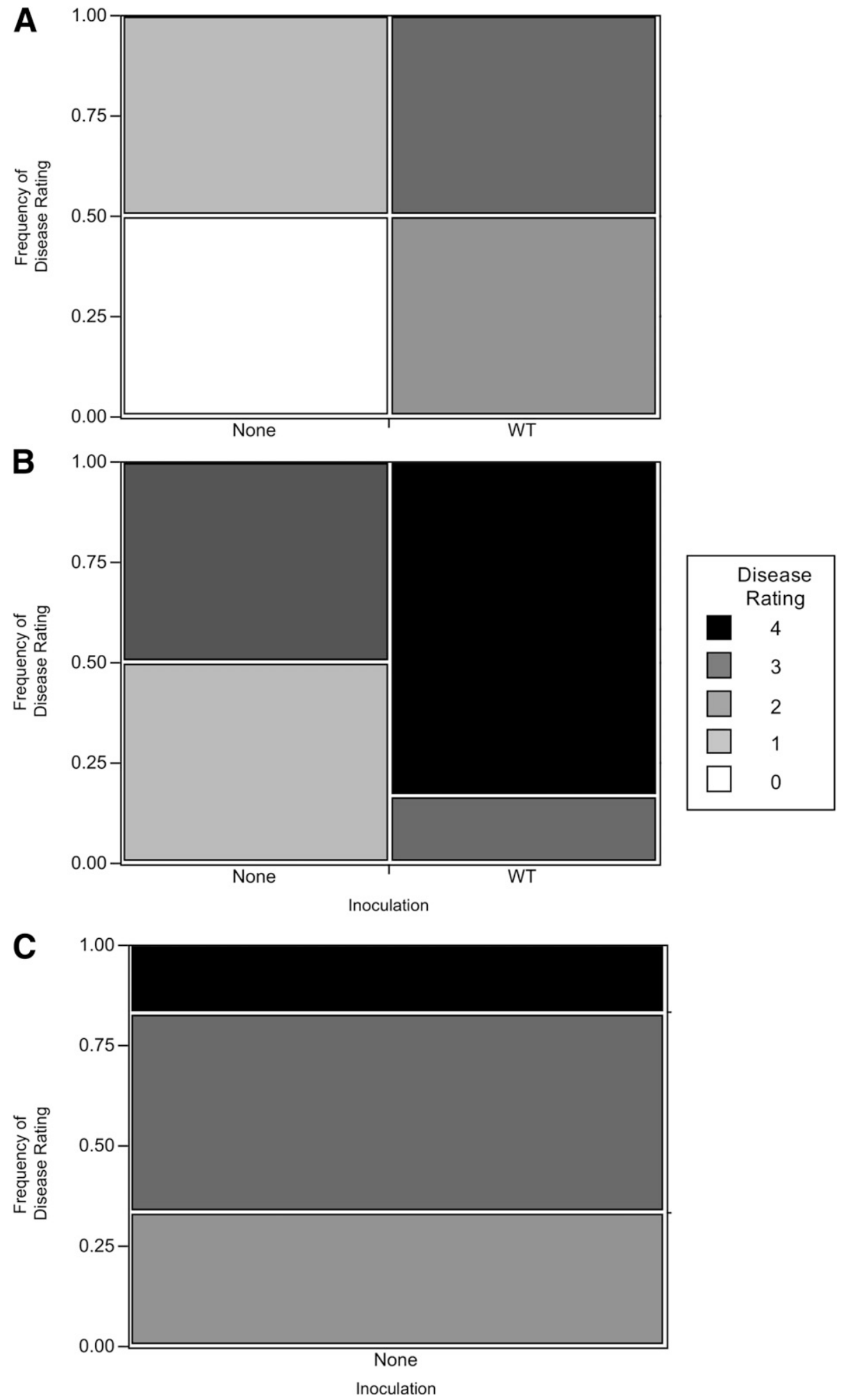

Fig. 9. Frequency of disease severity on individual Vitis vinifera 'Red Globe' grape berries either not inoculated or inoculated with a suspension of organisms previously recovered from wild-type (WT) Drosophila melanogaster fruit flies, the majority of which were in the orders Enterobacteriales and Saccharomycetales. These treatments were either $\mathbf{A}$, not exposed to insects; $\mathbf{B}$, exposed to axenic D. melanogaster; or $\mathbf{C}$, exposed to WT D. melanogaster during an 8-day incubation period. Disease severity is based on a 0 -to- 4 scale, where $0=$ berry is symptomless and completely intact; $1=$ berry is completely intact, with some discoloration of the skin only around the wound site; $2=$ berry is entirely intact but with obvious discoloration of the skin extending beyond the wound site; $3=$ majority of the berry skin is discolored but berry is still intact; and $4=$ berry is no longer intact, the inner pulp is liquefied and leaking, and the skin is completely discolored (characteristic sour rot symptoms). 
clusters also had Drosophila present within the bags as both larvae and adults, which apparently were introduced as unseen eggs or internal larvae at the start of the experiment. When all five clusters were symptomless initially, the four outer clusters developed symptoms of both Botrytis bunch rot and sour rot after 10 days of incubation (21 and 3\% severity levels, respectively). When the four outer clusters were initially symptomatic for Botrytis bunch rot and surrounded a healthy cluster, their Botrytis rot severity level increased from 50 to nearly $100 \%$ by the end of the incubation period, whereas only trace levels of sour rot developed in them (Fig. 10). When the center cluster was initially affected by sour rot, the previously healthy clusters surrounding it developed only a trace level of Botrytis bunch rot but $>50 \%$ sour rot severity. When center clusters were initially affected by sour rot and surrounded by Botrytis rot clusters, there was no postincubation change in the severity of Botrytis bunch rot in the surrounding clusters, although these clusters developed sour rot with a $30 \%$ severity rating (Fig. 10).

Disease development on a single cluster surrounded by four others was affected by both its own initial disease status and that of the surrounding clusters. A healthy cluster surrounded by healthy or Botrytis rot clusters developed $<10$ versus $>30 \%$ Botrytis bunch rot severity following the 10-day incubation period. Similar, relatively low levels of sour rot developed on initially healthy-center clusters regardless of whether or not the surrounding clusters were healthy or affected by Botrytis bunch rot (Fig. 11). An initial our rot severity of $59 \%$ increased to 70 to $80 \%$ when previously diseased clusters were surrounded by either healthy or Botrytis rot clusters, whereas little to no Botrytis bunch rot developed on these same center clusters. Significantly $(P<0.0001, t$ test $)$ higher levels of Botrytis bunch rot severity developed on initially healthy center clusters surrounded by other healthy clusters than on clusters initially affected with sour rot similarly surrounded by healthy clusters (Fig. 11).

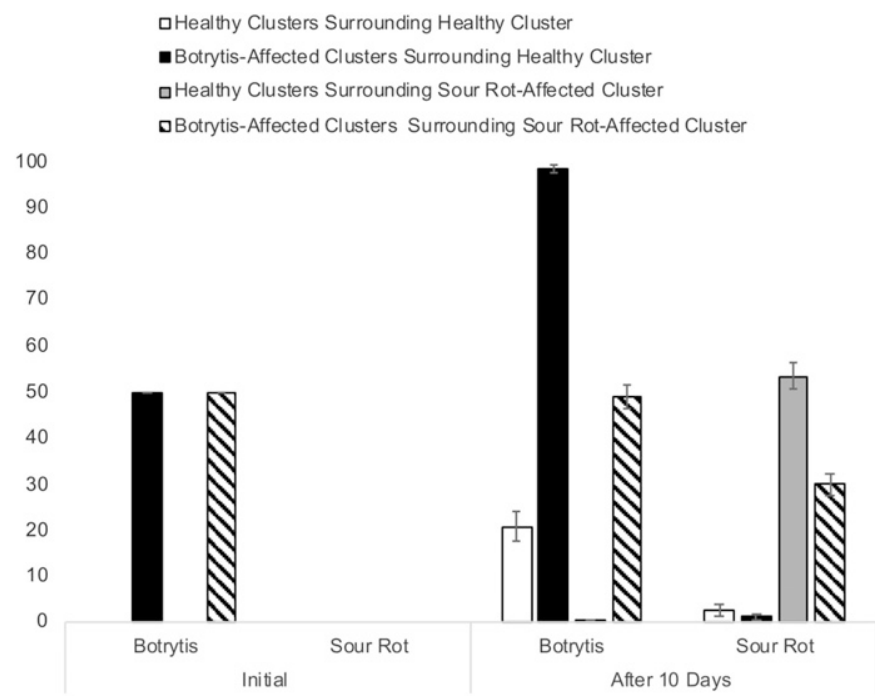

Fig. 10. Mean disease severity (percent cluster area symptomatic) of Botrytis bunch rot and sour rot on initially healthy or diseased field-collected grape clusters before and after 10 days of incubation within a plastic bag while surrounding one other initially healthy or diseased cluster. For clusters initially affected with Botrytis bunch rot or sour rot, the severity level of the indicated disease was $50 \%$ at the start of the experiment, whereas they were symptomless for the other. Within each bag, clusters were arranged in a pattern, with four clusters of one treatment forming a square surrounding the indicated cluster in the center. All clusters had 1 to $2 \mathrm{~cm}$ of space between them. Expressed values represent the means from 12 clusters per treatment, derived by combining the data from three runs of the experiment (two utilizing clusters of Vitis vinifera 'Riesling' and one utilizing Vitis interspecific hybrid cultivar Vignoles) in which there were four replicate clusters per treatment, after confirming their homogeneity of variance. Error bars represent the standard error of the mean.

\section{DISCUSSION}

Our results indicate that sour rot is caused by an interaction involving yeast, acetic acid bacteria, and Drosophila fruit flies, and that all three components must be present for typical symptoms to develop. It appears that multiple yeast genera are capable of producing the ethanol precursor of acetic acid within wounded berries. Our results and those of previous studies (Barata et al. 2008, 2012a,b; Bisiach et al. 1986; Guerzoni and Marchetti 1987) associate many different yeast species with diseased samples in the field. Our inoculation studies with selected species invariably resulted in ethanol production, and ethanol is a typical product of yeast metabolism. However, inoculations with yeast alone did not produce acetic acid in our experiments, which is consistent with the results of Barata et al. (2012a). Indeed, as postulated by Barata et al. (2012a), we did not obtain significant acetic acid production unless berries were coinoculated with both yeast and acetic acid bacteria. By assaying both ethanol and acetic acid production on a daily basis following such a coinoculation, we demonstrated that acetic acid was not produced within berries until ethanol was first produced (presumably, by the yeast) and that this final step (presumably, the result of bacterial activity) appeared to be catalyzed by the presence of Drosophila flies. In several preliminary experiments where we compared the contribution of D. melanogaster and D. suzukii (spotted-wing Drosophila) in inoculation experiments, we saw no difference between these two species in their influence on sour rot development (data not shown). Thus, because D. melanogaster is by far the more common of the two associated with diseased samples in the field (Hall 2018), we focused our studies on this species and suggest that our results are likely applicable to drosophilids in general.

We have defined sour rot as a syndrome consisting of both the production of acetic acid within necrotic berries and the loss of berry integrity resulting in the release of liquefied pulp, the latter characteristic also having been described by others as a defining one for sour rot (Barata et al. 2008; Bisiach et al. 1986; Guerzoni and

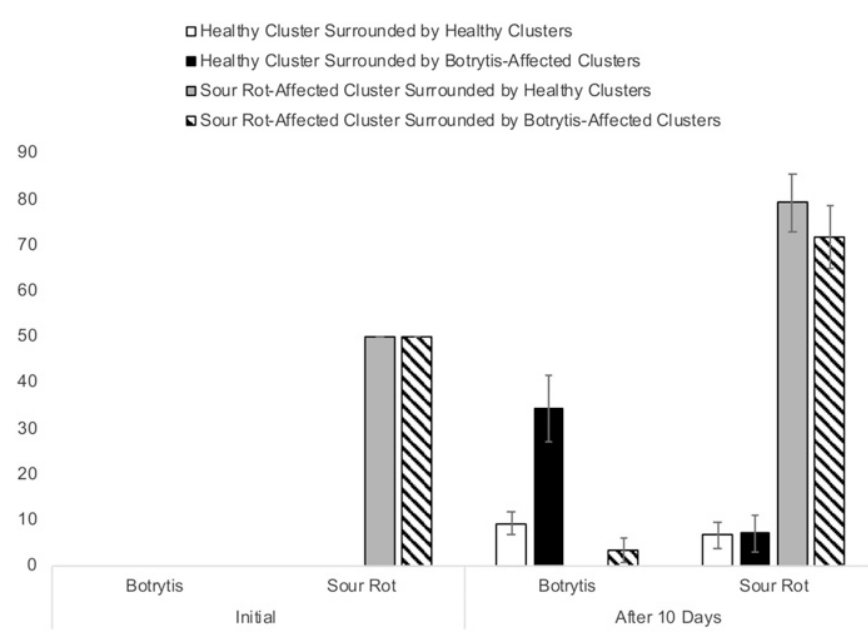

Fig. 11. Mean disease severity (percent cluster area symptomatic) of Botrytis bunch rot and sour rot on initially healthy or diseased field-collected grape clusters before and after 10 days of incubation within a plastic bag while surrounded by four healthy or diseased clusters. For clusters initially affected with Botrytis bunch rot or sour rot, the severity level of the indicated disease was $50 \%$ at the start of the experiment, whereas they were symptomless for the other. Within each bag, clusters were arranged in a pattern, with four clusters of one treatment forming a square surrounding the indicated cluster in the center. All clusters had 1 to $2 \mathrm{~cm}$ of space between them. Expressed values represent the means from 12 clusters per treatment, derived by combining the data from three runs of the experiment (two utilizing clusters of Vitis vinifera 'Riesling' and one utilizing Vitis interspecific hybrid cultivar Vignoles) in which there were four replicate clusters per treatment, after confirming their homogeneity of variance. Error bars represent the standard error of the mean. 
Marchetti 1987). Previous researchers determined that yeast and acetic acid bacteria were associated with sour rot (Barata et al. 2008, 2012 a,b; Bisiach et al. 1982, 1986; Guerzoni and Marchetti 1987) and, whereas some combinations of yeast and acetic acid bacteria successfully produced acetic acid in grape berries that we inoculated and resulted in their discoloration without exposure to Drosophila, a significant loss of berry integrity was not observed unless these insects were present. Loss of berry integrity, or turgor as Bisiach et al. (1986) termed it, is a crucial component in the final stage of sour rot. The associated leaking of microbe- and acetic-acid-filled pulp makes these microbes readily available for vectoring to new clusters by the adult drosophilids (or potentially other insects visiting the clusters, such as yellow jackets and other wasps) that are present, while also liberating acetic acid and ethanol volatiles that attract them (Barata et al. 2012a; Bisiach et al. 1986). Thus, drosophilids appear to be an integral component in both the development and spread of sour rot, as the disease has been defined here and by others (Barata et al. 2012a; Bisiach et al. 1986).

The disease name "sour rot" is sometimes used as a synonym for a syndrome also termed "summer bunch rot", caused by various filamentous fungi, including Aspergillus niger, Alternaria carbonarius, A. tenuis, B. cinerea, Cladosporium herbarum, Rhizopus arrhizus, and Penicillium spp. (Haviland et al. 2017). However, even though some of these organisms have been shown to be capable of decaying inoculated berries (Rooney-Latham et al. 2008), the same studies have not demonstrated the production of acetic acid in tandem with such decay. It seems possible that the yeast and bacteria responsible for ethanol and subsequent acetic acid production gain entry or become active in berries infected with these fungi after the berries begin to decay, subsequently producing the vinegar aroma noted in vineyards where these rots occur (Haviland et al. 2017). If so, the filamentous fungi might function as predisposing agents for sour rot under such circumstances, because wounds are generally acknowledged as necessary for disease development (McFadden-Smith and Gubler 2015), although this is purely speculative in the absence of supporting experimental data. Whereas we did not focus our attention on the potential role of filamentous fungi in sour rot development, our one set of experiments in which we inoculated only with Aspergillus niger produced no acetic acid or significant berry discoloration without the concurrent presence of wild-type $D$. melanogaster flies, which we showed harbor a suite of yeast and bacteria on and in their bodies that are capable of producing such symptoms themselves. Thus, we conclude that filamentous fungi are of secondary importance in this complex as we have studied it, as did Bisiach et al. (1982).

Barata et al. (2012a) concluded that the role of Drosophila spp. in sour rot development extends beyond that of a vector of microorganisms; specifically, they postulated that the insects prevent the grape berries from healing wounds because the proliferation of the insects is faster than the ability of the berry to heal. Although we did not investigate this specific suggestion, through our use of axenic D. melanogaster we did conclusively demonstrate that the flies play a critical nonmicrobial role in sour rot development, both by catalyzing the conversion of ethanol to acetic acid and through their role in promoting the loss of berry integrity. Although we did not examine the mechanisms underlying these phenomena, we believe they could involve, at least in part, enzymes released by the larval stage in order to facilitate consumption of the pulp (Gregg et al. 1990; Sakaguchi and Suzuki 2013). The precise mechanistic role of Drosophila involvement in sour rot development appears to provide a rich target for further research.

The ubiquitous presence of several genera of yeast or acetic acid bacteria (e.g., Saccharomyces, Pichia, Hanseniaspora, Metschnikowia, Acetobacter, and Gluconobacter) within healthy grape berries complicates the interpretation of the results from our pathogenicity experiments, and accounts for the presence of ethanol and acetic acid within many of our noninoculated control treatments, albeit at generally low levels. Although we tried several methods of sterilizing healthy berry pulp prior to inoculation (e.g., heat treatment), all efforts resulted in the disintegration of the berries, which then were not usable. Future research in which the interior of the grape is successfully sterilized or pasteurized without compromising the physical integrity of the cells (perhaps through irradiation) would obviate this confounding factor. To the best of our knowledge, the presence of these microorganisms as endophytes within healthy berries has not been reported previously, and has implications beyond those pertaining to sour rot (e.g., winemaking). Nevertheless, these results suggest that the primary inoculum necessary for sour rot development may, in some cases, be present endophytically. Relatedly, the oftennoted requirement for wounding may relate to processes beyond promoting the access of fruit flies and the microorganisms they carry to the berry interior (e.g., the release of cellular contents that provide substrates from which the appropriate introduced or endophytic microorganisms can produce ethanol and acetic acid and the ingress of oxygen required for the conversion of ethanol to acetic acid).

Bisiach et al. (1982) examined the relationship between Botrytis bunch rot and sour rot, concluding that existing Botrytis infections were halted in the presence of sour rot and that sour rot prevented the advancement of further fungal infection. Our experiments support this conclusion, because we found that Botrytis infections did not advance in the presence of a sour rot-infected cluster, whereas sour rot infections on clusters surrounded by Botrytis rot clusters continued to advance, although at a significantly slower rate than they would have if surrounded by healthy clusters. Thus, it appears that Botrytis rot and sour rot are competing for the same niche, because the presence of one impacted the rate of colonization by the other.

\section{ACKNOWLEDGMENTS}

We thank G. Brind' Amour for technical assistance in rearing Drosophila, D. Manns and P. Raes for conducting all HPLC runs, J. Burr for assistance in plating isolates, and I. O'Bryon for analysis of sequencing data.

\section{LITERATURE CITED}

Altschul, S. F., Gish, W., Miller, W., Myers, E. W., and Lipman, D. J. 1990. Basic local alignment search tool. J. Mol. Biol. 215:403-410.

Barata, A., Campo, E., Malfeito-Ferreira, M., Loureiro, V., Cacho, J., and Ferreira, V. 2011. Analytical and sensorial characterization of the aroma of wines produced with sour rotten grapes using GC-O and GC-MS: Identification of key aroma compounds. J. Agric. Food Chem. 59:2543-2553.

Barata, A., Correia Santos, S., Malfeito-Ferreira, M., and Loureiro, V. 2012a. New insights into the ecological interaction between grape berry microorganisms and Drosophila flies during the development of sour rot. Microb. Ecol. 64:416-430.

Barata, A., Gonzalez, S., Malfeito-Ferreira, M., Querol, A., and Loureiro, V. 2008. Sour rot-damaged grapes are sources of wine spoilage yeasts. FEMS Yeast Res. 8:1008-1017.

Barata, A., Malfeito-Ferreira, M., and Loureiro, V. 2012b. Changes in sour rotten grape berry microbiota during ripening and wine fermentation. Int. J. Food Microbiol. 154:152-161.

Bisiach, M., Minervini, G., and Salomone, M. C. 1982. Recherches expérimentales sur la pourriture acide de la grappe et sur ses rapports avec la pourriture grise 1. EPPO Bull. 12:15-27.

Bisiach, M., Minervini, G., and Zerbetto, F. 1986. Possible integrated control of grapevine sour rot. Vitis 25:118-128.

Broderick, N. A., Buchon, N., and Lemaitre, B. 2014. Microbiota-induced changes in Drosophila melanogaster host gene expression and gut morphology. MBio 5:e01117-14.

Bokulich, N. A., Thorngate, J. H., Richardson, P. M., and Mills, D. A. 2014. Microbial geography of wine grapes is conditioned by cultivar, vintage, and climate. Proc. Natl. Acad. Sci. USA 111:E139-148.

Caporaso, J. G., Bittinger, K., Bushman, F. D., DeSantis, T. Z., Andersen, G. L., and Knight, R. 2010a. PyNAST: A flexible tool for aligning sequences to a template alignment. Bioinformatics 26:266-267.

Caporaso, J. G., Kuczynski, J., Stombaugh, J., Bittinger, K., Bushman, F. D., Costello, E. K., Fierer, N., Pena, A. G., Goodrich, J. K., Gordon, J. I., Huttley, G. A., Kelley, S. T., Knights, D., Koenig, J. E., Ley, R. E., 
Lozupone, C. A., McDonald, D., Muegge, B. D., Pirrung, M., Reeder, J., Sevinsky, J. R., Turnbaugh, P. J., Walters, W. A., Widmann, J., Yatsunenko, T., Zaneveld, J., and Knight, R. 2010b. QIIME allows analysis of highthroughput community sequencing data. Nat. Methods 7:335-336.

Castellari, M., Versari, A., Spinabelli, U., Galassi, S., and Amati, A. 2001. An improved HPLC method for the analysis of organic acids, carbohydrates, and alcohols in grape musts and wines. J. Liq. Chrom. Rel. Technol. 23: 2047-2056.

Chandler, J. A., Lang, J. M., Bhatnagar, S., Eisen, J. A., and Kopp, A. 2011. Bacterial communities of diverse Drosophila species: Ecological context of a host-microbe model system. PLoS Genet. 7:e1002272.

DeSantis, T. Z., Hugenholtz, P., Larsen, N., Rojas, M., Brodie, E. L., Keller, K., Huber, T., Dalevi, D., Hu, P., and Andersen, G. L. 2006. Greengenes, a chimera-checked 16S rRNA gene database and workbench compatible with ARB. Appl. Environ. Microbiol. 72:5069-5072.

Edgar, R. C. 2010. Search and clustering orders of magnitude faster than BLAST. Bioinformatics 26:2460-2461.

Gregg, T. G., McCrate, A., Reveal, G., Hall, S., and Rypstra, A. L. 1990. Insectivory and social digestion in Drosophila. Biochem. Genet. 28:197-207.

Guerzoni, E., and Marchetti, R. 1987. Analysis of yeast flora associated with grape sour rot and of the chemical disease markers. Appl. Environ. Microbiol. 53:571-576.

Hall, M. E., Loeb, G. M. and Wilcox, W. F. 2018. Control of sour rot using chemical and canopy management techniques. Am. J. Enol. Vitic. 69:342-350.

Hall, M. E., and Wilcox, W. F. 2019. Identification and frequencies of endophytic microbes within healthy grape berries. Am. J. Enol. Vit. 70:2. (In press).

Hartigan, J. A., and Kleiner, B. 1981. Mosaics for contingency tables. Pages 268-273 in: Computer Science and Statistics: Proc. 13th Symp. Interface. W. F. Eddy, ed. Springer, New York.

Haviland, D. R., Bettiga, L. J., Varela, L. G., Roncoroni, J. A., Smith, R. J., Westerdahl, B. B., Bentley, W. J., Daane, K. M., Ferris, H., Gubler, W. D., Hembree, K. J., Ingels, C. A., Zalom, F. G., and Zasada, I. 2017. UC IPM Pest Management Guidelines Grape. UC ANR Publ. 3448. Online publication. Oakland, CA. http://ipm.ucanr.edu/PMG/pmgauthors.html?grapes
Kõljalg, U., Nilsson, R. H., Abarenkov, K., Tedersoo, L., Taylor, A. F. S., Bahram, M., Bates, S. T., Bruns, T. D., Bengtsson-Palme, J., Callaghan, T. M., Douglas, B., Drenkhan, T., Eberhardt, U., Dueñas, M., Grebenc, T., Griffith, G. W., Hartmann, M., Kirk, P. M., Kohout, P., Larsson, E., Lindahl, B. D., Lücking, R., Martín, M. P., Matheny, P. B., Nguyen, N. H., Niskanen, T., Oja, J., Peay, K. G., Peintner, U., Peterson, M., Põldmaa, K., Saag, L., Saar, I., Schüßler, A., Scott, J. A., Senés, C., Smith, M. E., Suija, A., Taylor, D. L., Telleria, M. T., Weiss, M., and Larsson, K.-H. 2013. Towards a unified paradigm for sequence-based identification of fungi. Mol. Ecol. 22: 5271-5277.

Koyle, M. L., Veloz, M., Judd, A. M., Wong, A. C., Newell, P. D., Douglas, A. E., and Chaston, J. M. 2016. Rearing the fruit fly Drosophila melanogaster under axenic and gnotobiotic conditions. J. Vis. Exp. 113:e54219. doi:link

McFadden-Smith, W., and Gubler, W. D. 2015. Sour Rot. Pages 87-90 in: Compendium of Grape Diseases, Pests, and Disorders, 2nd ed. W. F. Wilcox, W. D. Gubler, and J. K. Uyemoto, eds. American Phytopathological Society Press, St. Paul, MN.

Parks, D. H., Tyson, G. W., Hugenholtz, P., and Beiko, R. G. 2014. STAMP: Statistical analysis of taxonomic and functional profiles. Bioinformatics 30: 3123-3124.

Rooney-Latham, S., Janousek, C. N., Eskalen, A., and Gubler, W. D. 2008. First report of aspergillus carbonarius causing sour rot of table grapes (Vitis vinifera) in California. Plant Dis. 92:651.

Sakaguchi, H., and Suzuki, M. G. 2013. Drosophila melanogaster larvae control amylase secretion according to the hardness of food. Front. Physiol. 4:200.

Staubach, F., Baines, J. F., Kunzel, S., Bik, E. M., and Petrov, D. A. 2013. Host species and environmental effects on bacterial communities associated with Drosophila in the laboratory and in the natural environment. PLoS One 8: e70749.

Wong, C. N., Ng, P., and Douglas, A. E. 2011. Low-diversity bacterial community in the gut of the fruitfly Drosophila melanogaster. Environ. Microbiol. 13:1889-1900. 